\title{
Quantitative Analysis of Synaptic Vesicle Rabs Uncovers Distinct Yet Overlapping Roles for Rab3a and Rab27b in $\mathrm{Ca}^{2+}$-Triggered Exocytosis
}

\author{
Nathan J. Pavlos, ${ }^{1}$ Mads Grønborg, ${ }^{1}$ Dietmar Riedel, ${ }^{2}$ John J. E. Chua, ${ }^{1}$ Janina Boyken, ${ }^{1}$ Tobias H. Kloepper, ${ }^{3}$ \\ Henning Urlaub, ${ }^{4}$ Silvio 0. Rizzoli, ${ }^{5}$ and Reinhard Jahn ${ }^{1}$ \\ ${ }^{1}$ Department of Neurobiology and Laboratories of ${ }^{2}$ Electron Microscopy, ${ }^{3}$ Structural Biochemistry, and ${ }^{4}$ Bioanalytical Mass Spectrometry, Max Planck \\ Institute for Biophysical Chemistry, 37077 Göttingen, Germany, and ${ }^{5}$ European Neuroscience Institute, 37077 Göttingen, Germany
}

Rab GTPases are molecular switches that orchestrate protein complexes before membrane fusion reactions. In synapses, Rab3 and Rab5 proteins have been implicated in the exo-endocytic cycling of synaptic vesicles (SVs), but an involvement of additional Rabs cannot be excluded. Here, combining high-resolution mass spectrometry and chemical labeling (iTRAQ) together with quantitative immunoblotting and fluorescence microscopy, we have determined the exocytotic (Rab3a, Rab3b, Rab3c, and Rab27b) and endocytic (Rab4b, Rab5a/b, Rab10, Rab11b, and Rab14) Rab machinery of SVs. Analysis of two closely related proteins, Rab3a and Rab27b, revealed colocalization in synaptic nerve terminals, where they reside on distinct but overlapping SV pools. Moreover, whereas Rab3a readily dissociates from SVs during $\mathrm{Ca}^{2+}$-triggered exocytosis, and is susceptible to membrane extraction by Rab-GDI, Rab27b persists on SV membranes upon stimulation and is resistant to GDI-coupled Rab retrieval. Finally, we demonstrate that selective modulation of the GTP/GDP switch mechanism of Rab27b impairs SV recycling, suggesting that Rab27b, probably in concert with Rab3s, is involved in SV exocytosis.

\section{Introduction}

Synaptic vesicles (SVs) are storage organelles for neurotransmitters that undergo $\mathrm{Ca}^{2+}$-triggered exocytosis upon arrival of an action potential at the synaptic nerve terminal. Following exocytotic membrane fusion, SV constituents are retrieved by endocytosis. While the fine details of the endocytotic trafficking routes remain controversial, clathrin-mediated endocytosis and early endosomes are likely involved as intermediates (Rizzoli and Betz, 2004; Südhof, 2004). Exocytosis of SVs is mediated by an array of evolutionarily conserved synapse-specific proteins that orchestrate vesicle attachment to the plasma membrane (docking), activation of the fusion machinery (priming), and finally membrane fusion. Whereas SNAREs (soluble $N$-ethylmaleimide-sensitive factor attachment protein receptors) and SM (Sec1/Munc18-like) proteins are widely recognized as the minimal fusogenic machinery (Jahn and Scheller, 2006), small GTPases of the Rab family are

\footnotetext{
Received Feb. 18, 2010; revised July 19, 2010; accepted Aug. 11, 2010.

N.J.P. is supported by a National Health and Medical Research Council (Australia) CJ Martin Fellowship (ID: 463911). M.G. is supported by a research stipend from the Alfred Benzon Foundation. This work was supported in part by a grant from the European Union commission (ProjectEU-Synapse, FP6). We are indebted to Drs. Francis Barr Marino Zerial, Mika Simons, Jack Francis, Arnard Echard, Henrik Martens, and Miguel Seabra for the generous provision of CDNA constructs and antibodies. We thank Dr. Ulf Geumann and Ina Herefort for the preparation of recombinant His-GDI and neuron cultures.

Correspondence should be addressed to Reinhard Jahn, Department of Neurobiology, Max Planck Institute for Biophysical Chemistry, 37077 Göttingen, Germany. E-mail: rjahn@gwdg.de.

N. J. Pavlos's present address: Centre for Orthopaedic Research, School of Surgery, University of Western Australia, Nedlands, WA 6009, Australia.

T. H. Kloepper's present address: MRC Laboratory of Molecular Biology, Hills Road, Cambridge CB2 OQH, UK.

DOI:10.1523/JNEUROSCI.0907-10.2010

Copyright $\odot 2010$ the authors $\quad 0270-6474 / 10 / 3013441-13 \$ 15.00 / 0$
}

thought to confer the specificity of membrane targeting in intracellular fusion reactions (Zerial and McBride, 2001; Pfeffer and Aivazian, 2004).

Over $60 \mathrm{Rab}$ proteins are known to exist in humans, each thought to localize to a distinct subcellular organelle. Like other GTPases, Rabs function as molecular switches, oscillating between GTP-bound (active) and GDP-bound (inactive) conformations with cycles of membrane association/dissociation. This "on/off" cycling enables Rabs to reversibly recruit GTPdependent effectors through which they elicit their regulatory functions at multiple stages of vesicular transport. Along with their intrinsic GTPase activity, Rab activation is regulated by the antagonistic activities of Rab guanine nucleotide exchange factors (GEFs) and Rab GTPase-activating proteins (GAPs), which catalyze Rab GDP/GTP exchange and nucleotide hydrolysis. Following GTP uncoupling, GDP-bound Rabs are retrieved from membranes by soluble guanine nucleotide dissociation inhibitors (GDIs), which stabilize cytosolic Rabs until reactivated for a new round of membrane cycling (Grosshans et al., 2006; Stenmark, 2009).

Despite a wealth of knowledge regarding the mechanisms by which Rabs regulate intracellular docking and fusion events, it has been surprisingly difficult to reconcile the functions of Rabs in SV exocytosis and recycling. To some extent, this probably reflects the redundancy that exists between Rab family members (Pereira-Leal and Seabra, 2000). For instance, Rab3, the most abundant of all Rabs in the brain (Schlüter et al., 2002), is represented by four homologous isoforms $(\mathrm{Rab} 3 \mathrm{a} / \mathrm{b} / \mathrm{c} / \mathrm{d})$. However, although long considered central to the SV exocytotic release 
mechanism, quadruple knock-out of all four Rab3 isoforms results in only a modest reduction $(\sim 30 \%)$ in evoked synaptic response (Schlüter et al., 2004). Similar mild synaptic defects are also observed in Rab3 deletion mutants of Caenorhabditis elegans, which possesses a single rab-3 allele (Nonet et al., 1997). A few additional insights have also been gained from the functional dissection of Rab3 effectors, including Rabphilin and RIM, both proving dispensable for Rab3 targeting and function following genetic ablation (Schlüter et al., 1999; Castillo et al., 2002).

While the role of Rab3 proteins in SV cycling remains incompletely understood, other Rabs have been proposed to function in presynaptic trafficking. Although it is still uncertain whether endosomes and their associated Rab repositories are involved as mandatory intermediates in every SV recycling event, the endosomal Rab5 GTPase has been shown to be involved in SV recycling in flies and mice (Shimizu et al., 2003; Wucherpfennig et al., 2003; Star et al., 2005). Furthermore, both Rab11b (Khvotchev et al., 2003) and Rab27 (Mahoney et al., 2006; Yu et al., 2008) have been recently suggested to play a role in the synapse, but their SV localizations and function(s) have yet to be assigned.

To further our understanding of Rabs in presynaptic membrane traffic, an important first step is to unequivocally identify all Rabs that are localized to SVs. Only once the complete SV Rab inventory is known will it be possible to sort out potential redundancies and unambiguously determine which Rabs function in the trafficking steps of the SV cycle. Recent proteomic analysis of SVs, however, revealed a surprising multitude of Rab proteins (more than half of the genetic inventory), including some that so far have been assigned to completely different trafficking steps (such as Rab1, whose function in ER-to-Golgi trafficking is firmly established) (Takamori et al., 2006), thus hinting that a lot more Rabs function in the SV cycle than hitherto assumed.

Here, in an attempt to quantitatively refine the identities and relative abundance of Rab proteins on SVs, we have combined state-of-the-art proteomics with immunoanalytical and microscopy-based methods. Based on these analyses, we now show that only a subset of Rabs originally identified on SV membranes is indeed highly enriched on SVs, encompassing both exocytotic and endosomal elements. We propose that these GTPases may constitute the core "Rab" machinery underscoring exoendocytic SV cycling and discuss their possible involvement at neuronal synapses with particular emphasis on two closely related proteins, Rab3a and Rab27b.

\section{Materials and Methods}

Antibodies. Refer to supplemental Table 1 (available at www. jneurosci.org as supplemental material) for a complete list of Rab antibodies, their sources, and immunoblot and immunofluorescence efficiencies. Antibodies against synaptophysin (1:10,000), synaptobrevin/VAMP2 (1:10,000), GDI (Cl 81.2) (1:1000), NMDAR (1:1000), GFP (1:5000), and MAP2 (1:3000) were obtained from Synaptic Systems. EEA1 (1:1000) and GM130 (1:1000) were purchased from BD Biosciences. $\mathrm{Na}^{+} / \mathrm{K}^{+}$-ATPase $(1: 1000)$ was from Abcam. Fluorophoreconjugated (Cy2, Cy3, Cy5, and AMCA) secondary antibodies (1:100 to 1:500) were from Jackson ImmunoResearch Laboratories. Horseradish peroxidase-conjugated secondary antibodies $(1: 2000$ to $1: 10,000)$ were purchased from Bio-Rad.

Plasmids. N-terminal pEGFP and pmRFP chimeras of Rab3A were generated by subcloning a PCR-amplified Rab3A open reading frame from mouse brain into either an EGFP (Clontech) or mRFP vectors. pEGFP-Rab1b, -Rab6b, -Rab8b, -Rab9, -Rab10, -Rab11b, and -Rab14 were generous gifts from Francis Barr (University of Liverpool, Liverpool, UK). pEGFP-Rab5a and His-GDI were kind gifts from Marino Zerial [Max Planck Institute (MPI) for Cell Biology and Genetics,
Dresden, Germany]; pEGFP-Rab7 was a gift from Bo van Deurs (University of Copenhagen, Copenhagen, Denmark); pEGFP-Rab35 was kindly donated by Mika Simons (MPI for Experimental Medicine, Göttingen, Germany); pEGFP-Rab18 was provided by Dr. Fraser Coxon (University of Aberdeen, Aberdeen, UK); Rab27bQ78L, Rab27bT23N, and Rab27bN133I cDNAs subcloned into the pEGFP vectors were kindly provided by Dr. Miguel Seabra (Imperial College of London, London, UK) and pEGFP-Rab4b was kindly provided by Dr. Henrik Martens (Synaptic Systems, Göttingen, Germany).

Isolation of synaptic organelles from rat brain homogenates. The methodologies for the isolation and characterization of highly purified rat SVs are based on our previously published protocols (Takamori et al., 2006). Synaptosomes were purified from rat brain as previously described (Fischer von Mollard et al., 1991). For subcellular fractionation studies, osmotically lysed synaptosomes were overlaid on a $0.4-1.2 \mathrm{M}$ continuous sucrose gradient and centrifuged at $100,000 \times g$ for $1 \mathrm{~h}$. The gradients were fractionated from the bottom, and equal volumes of gradient fractions were analyzed by immunoblotting.

Digestion and ITRAQ labeling. Rat brain homogenates were digested in-solution by trypsin, and tryptic peptides were then tagged with iTRAQ reagent according to the manufacturer's instructions (iTRAQ reagent kit, Applied Biosystems). Brain homogenates corresponding to fractions starting brain homogenate (H), synaptic cytosol (S3), crude SVs (LP2), and highly purified SVs (SV) were tagged with iTRAQ reagents 114, 115, 116, or 117, respectively (see Fig. $1 b$ ).

Strong cation exchange fractionation. Following digestion, samples were fractionated off-line on an ICAT strong cation exchange (SCX) column (Applied Biosystems) according to the manufacturer's instructions. Peptides were then eluted stepwise by adding $500 \mu \mathrm{l}$ of $\mathrm{KCl}$ solutions of increasing concentration $(5 \mathrm{~mm}, 100 \mathrm{mM}, 150 \mathrm{mM}, 200 \mathrm{~mm}, 300$ $\mathrm{mm}, 400 \mathrm{~mm}, 500 \mathrm{~mm}, 600 \mathrm{~mm}, 800 \mathrm{~mm}$, and $1000 \mathrm{~mm}$ ) in $10 \mathrm{~mm}$ $\mathrm{KH}_{2} \mathrm{PO}_{4}, 25 \%$ acetonitrile, $\mathrm{pH}$ 3.0. The samples were desalted on a custom built micro-column with POROS Oligo R2 RP material.

Mass spectrometry and quantification. All SCX fractions were analyzed on a Thermo LTQ XL Orbitrap (Thermo Fisher Scientific) coupled to an Agilent 1100 series LC-5 system (Agilent Technologies). Peptides were separated at a flow rate of $200-300 \mathrm{nl} / \mathrm{min}$ on a custom-designed reversed phase column (C18, Reprosil, Maisch). Elution of peptides was performed with a 118 min gradient from $7.5-40 \%$ mobile phase B $(80 \%$ acetonitrile, $0.15 \%$ formic acid). Peak lists were searched against NCBI RefSeq database using Mascot v.2.2.04 as search engine. Mass accuracy was $10 \mathrm{ppm}$ for the parent ion and $30 \mathrm{ppm}$ for fragment ions. The peptides were constrained to be tryptic with a maximum of 2 missed cleavages. Carbamidomethylation of cysteines was considered a fixed modification, whereas oxidations of methionine residues were considered as variable modification. Quantification was performed using Mas$\cot \mathrm{v}$ 2.2.04. The protein ratio was calculated as a weighted median ratio where only unique (nonredundant) peptides with scores $>15$ were used for quantification.

Immunoblot analysis. SDS-PAGE and immunoblotting were performed according to the standard methods. Protein concentrations were determined using Bradford (Bio-Rad) or with the BCA method (Pierce). Quantification of immunoblots was performed using an in-house custom routine for Matlab (The MathWorks) as detailed by Bethani et al. (2007). The relative enrichment ratio was calculated by normalizing the intensity of each subcellular fraction against the intensity of starting rat brain homogenate.

Immunoisolation of SVs and Rab-GDI complexes. For SV immunoisolation, Immunobeads (Eupergit C1Z methacrylate microbeads; Röhm Pharmaceuticals) coupled to monoclonal antibodies C1 42.2 (Rab3A) were prepared and used as described previously (Fischer von Mollard et al., 1994a). Bound SVs were eluted by incubating the beads with sample buffer and resolved by SDS-PAGE and immunoblotting. For affinity chromatography of GDI-Rab complexes, GDI antibodies (Cl 81.2) were covalently coupled to protein G Sepharose (GE Healthcare), packed into a column, and incubated with concentrated rat brain presynaptic cytosol (LS2) in buffer A (10 mM HEPES buffer, pH 7.4, containing $100 \mathrm{~mm}$ $\mathrm{NaCl}, 320 \mathrm{~mm}$ sucrose) plus protease inhibitors for $2 \mathrm{~h}$ at $4^{\circ} \mathrm{C}$ on a rotating wheel. Samples were washed five times with buffer A, eluted with 
glycine (0.1 M, pH 2.5), and analyzed by immunoblotting and mass spectrometry.

Glutamate release, Rab dissociation, and Rab-GDI extraction assays. Isolation, stimulation, and subfractionation of synaptosomes from rat brain were performed according to Fischer von Mollard et al. (1991). Glutamate release was monitored using the photometric assay. Reduction of NADP was monitored at $360 \mathrm{~nm}$ (reference wavelength $390 \mathrm{~nm}$ ) using an Aminco DW 2000 spectrophotometer (Olis), using $1 \mathrm{mg}$ aliquots of synaptosomes, in $1 \mathrm{ml}$ of sodium buffer to which were added 1.3 mM calcium chloride, or $5 \mathrm{~mm}$ EGTA, $50 \mathrm{~mm} \mathrm{KCl}$, and $200 \mathrm{U}$ of glutamate dehydrogenase (Sigma). The total assay duration was $\sim 1 \mathrm{~h}$ following the isolation of synaptosomes (i.e., $10 \mathrm{~min}$ preincubation at $37^{\circ} \mathrm{C}$; glutamate release $10 \mathrm{~min} ; 1 \times 10 \mathrm{~min}$ centrifugation at 55,000 rpm; $1 \times 10 \mathrm{~min}$ centrifugation at $17,000 \mathrm{rpm} ; 1 \times 20 \mathrm{~min}$ centrifugation at 70,000 rpm). Rab extraction assays were performed essentially as detailed in the methods of Chou and Jahn (2000). Bacterial expression (BL21) was performed using the T7 polymerase hexa-histidine-tagged GDI expression vector and recombinant proteins purified over nickel-nitrilotriacetic acid agarose (Qiagen). Proteins were dialyzed and used routinely at standard concentrations $(0.2-2 \mu \mathrm{M})$.

Electron microscopy and immunogold labeling. Rat brain homogenates and highly purified SVs were absorbed to formvar-coated grids and counterstained with $1 \%$ uranylacetate. For immunogold labeling, the bound samples were fixed with $4 \%$ paraformaldehyde (PFA), quenched with $20 \mathrm{~mm}$ glycine, and immunostained using the described sera, followed by addition of Protein A-gold $(10 \mathrm{~nm})$. The preparations were then washed repeatedly with TPBS and high-salt TPBS $(0.5 \mathrm{M} \mathrm{NaCl})$ and postfixed with $2 \%$ glutaraldehyde. Following counterstaining with $1 \%$ uranylacetate samples were examined using a CM120 Philips electron microscope equipped with a TemCam 224A slow scan CCD camera (TVIPS). Electron microscopy of Immunobeads was performed as previously described (Takamori et al., 2000).

Neuronal culture, transfections, immunofluorescence, and ultrathin sectioning. Experiments were performed on primary hippocampal cultures from E18 rats and maintained in culture from 10 to $21 \mathrm{~d}$ in vitro (DIV). Neurons were grown in Neurobasal media supplemented with penicillin/ streptomycin, L-glutamine, B-27, and N-2 supplements (Invitrogen). At 7-10 DIV, neurons were transfected using the Profection mammalian transfection system-calcium phosphate (Promega) according to the manufacturer's instructions. Neurons were routinely transected with 10 $\mu \mathrm{g}$ of corresponding cDNAs and analyzed $48 \mathrm{~h}$ after transfection. Immunostaining of primary rat hippocampal cultures was performed according to standard methods. Briefly, neurons (10-17 DIV) were fixed for 20 $\mathrm{min}$ in $3 \%(\mathrm{w} / \mathrm{v})$ PFA, quenched for 10 min with $50 \mathrm{~mm}$ ammonium chloride, and permeabilized with $0.1 \%(\mathrm{v} / \mathrm{v})$ Triton X-100 for $5 \mathrm{~min}$ to allow labeling of internal cell structures. All solutions were made in PBS, and antibody staining was performed for $60 \mathrm{~min}$ at room temperature. In some instances, immunostained slides were postfixed with $2 \%$ glutaraldehyde and embedded in 2,4,6-tris[bis(methoxymethyl)amino]-1,3,5triazine (TCI Europe), and ultrathin sections $(\sim 100 \mathrm{~nm})$ were cut before sections were mounted for analysis by confocal microscopy (Leica Microsystems).

Image acquisition and processing and cross-correlation analysis. Images were routinely collected using an epifluorescence microscope (Axiovert 200M; Carl Zeiss MicroImaging) with either a $40 \times($ NA 1.2) or a $63 \times$ (NA 1.4) plan Apochromat oil-immersion objective (Carl Zeiss MicroImaging), standard filter sets (Carl Zeiss MicroImaging), a $1317 \times 1035$ pixel cooled CCD camera (Princeton Instruments), and MetaMorph software. Confocal microscopy was performed on a TCS SP5 confocal microscope (Leica Microsystems), using a 1.4 NA 100× objective (Leica Microsystems). For live-cell imaging, neurons were maintained at $37^{\circ} \mathrm{C}$ on a temperature-controlled chamber and time-lapse series acquired every $5 \mathrm{~s}$ up to $5 \mathrm{~min}$ using MetaMorph software (MetaMorph). Image stacks were processed either in ImageJ (NIH) or CS2 software (Adobe) without contrast or other adjustments, sized, and placed using Illustrator 11.0 (Adobe). Cross-correlation analysis and quantitation of image immunointensities was conducted using either custom-written routines for Matlab (The MathWorks) as described in detail by Bethani et al. (2007) or online ImageJ macros (NIH). Maximum correlation values (Pear- son's, $R r$ ) were calculated from samples transfected with EGFP-Rab3a and then counterstained for endogenous Rab3a (mean $R r=0.956$ ), and random values were calculated from these same images in which one image channel was flipped as a mirror image to the other $(R r=0.046)$.

FM4-64FX uptake studies. For SV labeling studies, transfected rat hippocampal neurons grown on coverslips were mounted on a custom-built chamber and action potentials were evoked using a platinum plate electrode (field stimulator); brief shocks $(100 \mathrm{~mA})$ were delivered using an A385 stimulus isolator and an A310 Accupulser stimulator (World Precision Instruments). SVs were labeled with the fixable fluorescent lipid membrane marker FM4-64FX N-[3-triethylammoniumpropyl)-4-(6(4-diethylamino) phenyl) hexatrienyl) pyridinium dibromide] by bath application of cells in neuronal buffer (124 mM NaCl, $5 \mathrm{~mm} \mathrm{KCl,} 2 \mathrm{~mm}$ $\mathrm{CaCl}_{2}, 1 \mathrm{~mm} \mathrm{MgCl}$, $30 \mathrm{~mm}$ glucose, $25 \mathrm{~mm}$ HEPES, pH 7.4) containing FM4-64FX $(10 \mu \mathrm{M})$ and delivering an electrical stimulation of 600 action potentials at $20 \mathrm{~Hz}$. Following stimulation, the cells were incubated in the presence of FM4-64FX for 90 s to ensure completion of endocytosis and then washed with dye-free neuronal buffer to remove unbound surface dye (10 min). Following fixation (4\% PFA, $20 \mathrm{~min}$ ), EGFP-labeled varicosities that colocalized with FM4-64FX were imaged by confocal microscopy and quantified from multiple randomized cells/ fields corresponding to hundreds of labeled synaptic boutons. Active synapses from nontransfected neurons were measured in parallel. Quantification of FM4-64FX was performed using custom-written routines in Matlab (The MathWorks).

\section{Results \\ Exocytotic and endosomal Rabs are specifically enriched on purified SVs}

To provide a quantitative assessment of the SV Rab proteome (Rabome), we used two complementary approaches to monitor copurification of Rab proteins with SVs during subcellular fractionation of rat brain. First, a nonbiased proteomic strategy was implemented using isobaric tag for relative quantitation (iTRAQ) together with liquid chromatography tandem mass spectrometry (LC-MS) (Fig. 1). For this purpose, we harvested select subcellular fractions (H, S3, and LP2) and highly purified SVs, the homogeneity and purity of which were confirmed by morphometry at the electron microscopy (EM) level (Fig. 1a) and marker protein analysis (supplemental Fig. 1, available at www.jneurosci.org as supplemental material). A complete overview of the rat brain fractionation and SV purification protocol is detailed in supplemental Figure 1 (available at www.jneurosci. org as supplemental material).

The corresponding rat brain homogenates were subjected to in-solution trypsin digestion and labeled with the indicated iTRAQ reagents (Fig. 1b). Isobaric iTRAQ reagents form covalent adducts to free $\mathrm{N}$ termini and lysine side chains and give rise to differential reporter ions (i.e., $m / z 114,115,116$, and 117) upon peptide fragmentation during the MS analysis. With this procedure, the relative abundance of each Rab protein in the various rat brain homogenates can be accurately quantified. Representative MS spectra of iTRAQ-labeled peptides from Rab3a and Rab18 are illustrated in Figure $1 b$. Protein quantification was performed using only unique (nonredundant) peptides to ensure unequivocal identification of each protein, which limited the analysis to 22 Rabs (Fig. 1c). Two independent biological replicates with at least two technical replicates each were conducted for the quantitative MS-analysis. The names, accession numbers, and iTRAQ ratios of all quantifiable Rabs are summarized in Table 1.

As an independent approach, we also performed quantitative immunoblotting of subcellular fractions obtained during the purification of SVs and probed for the enrichment of Rab proteins using a panel of Rab-specific antibodies (Fig. $2 a, b$; for antibody 
details, refer to supplemental Table 1, available at www.jneurosci.org as supplemental material).

With a few notable exceptions (e.g., Rab4), the data of the two quantitative approaches agreed closely with each other, allowing the following conclusions to be drawn. First, almost all of the Rabs showed some enrichment in the SV fraction (up to 4-fold, which was therefore defined as threshold, see below). This enrichment probably reflects Rab deposition from a GDI-soluble pool during SV isolation, and we therefore consider it as nonspecific. Second, a subset of Rab proteins was enriched to a significantly higher degree in the SV fraction than the other Rabs. As expected, this includes members of the Rab3 subfamily (Rab3a/b/c), with a $>10$-fold enrichment on purified SV that parallels that of bona fide SV membrane proteins such as synaptophysin and synaptobrevin (Fig. 2). Intriguingly, a similarly high enrichment was found for Rab27b using immunoblotting. In contrast, its closely related homolog Rab27a was not detectable (Fig. 2a). Phylogenetic analysis (supplemental Fig. 2, available at www.jneurosci.org as supplemental material) revealed that Rab27b shares closest homology with members of the Rab3 family (LM/AU: 0.81/0.84), explaining that it was missed in the MS analysis due to the lack of nonredundant peptides.

Third, we identified several Rabs that were enriched above threshold but to a lower degree than the SV-specific proteins. Interestingly, most of them have been implicated in the function of early and recycling endosomes. These include Rab5a/b isoforms, which occupy early endosomes but have also been localized to SVs (de Hoop et al., 1994; Fischer von Mollard et al., 1994b); Rab10 ( 6.3-fold), a relatively uncharacterized $\mathrm{Rab}$ protein thought to function in endosome recycling (Babbey et al., 2006); Rab11b $(\sim 4.3$-fold $)$, reported to localize to both perinuclear recycling endosomes (Ullrich et al., 1996) and SVs (Khvotchev et al., 2003); and Rab14 ( 10-fold), thought to function in the biosynthetic/recycling pathway between Golgi and endosomal membranes (Junutula et al., 2004). Note that two biosynthetic ER-Golgi Rabs, namely Rab1b (Plutner et al., 1991) and Rab2a (Tisdale and Balch, 1996), were enriched slightly beyond our cutoff value on SV membranes by iTRAQ/MS but not significantly above threshold in the Western blot analysis.

In summary, three distinct distribution patterns can be distinguished for Rab proteins: those showing strong copurification with SV markers, i.e., type I Rabs: Rab3 isoforms and Rab27b; moderate coenrichment type II Rabs: Rab4, Rab5, Rab7, Rab10, Rab11b, and Rab14; and weak coenrichment type III Rabs:

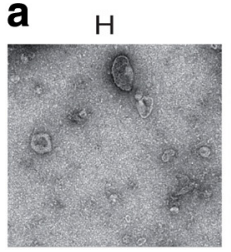

b
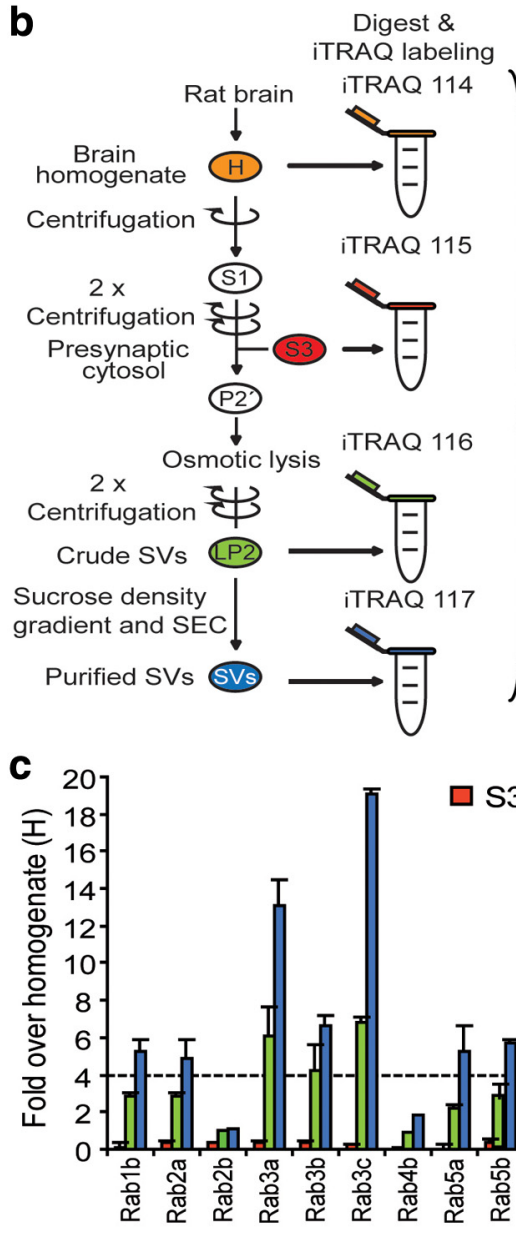

SV

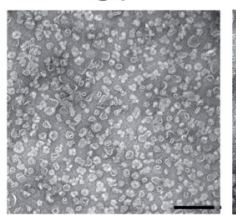

Mixed samples

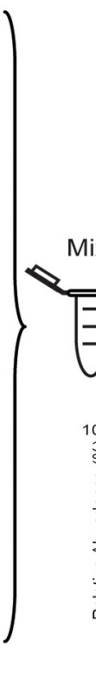

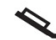
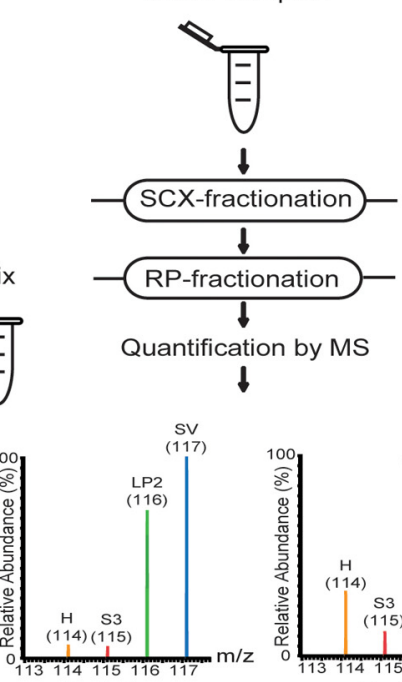

Rab3a

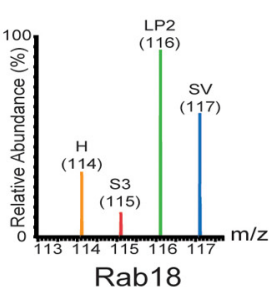

Figure 1. Identification and quantitation of Rab GTPases in rat brain homogenates by ITRAQ labeling and tandem MS. $\boldsymbol{a}$, EM characterization of negatively stained samples of starting brain homogenate, synaptic cytosol, crude SVs, and highly purified SVs. Scale bar, $0.5 \mu \mathrm{m} . \boldsymbol{b}$, A schematic of the rat SV purification protocol. Rat brains were homogenized and then centrifuged in two low-speed steps to generate a crude synaptosomal particulate fraction (P2') and cytosolic fraction (S3). Following osmotic lysis of the synaptosomes and two low-speed centrifugations, crude SVs were further purified by sucrose density centrifugation and size-exclusion chromatography (SEC) on controlled pore glass beads (CPG). Peak fractions of SVs were pooled and collected by centrifugation to yield a highly purified SV fraction. Fractions were digested with trypsin and the peptides labeled with 114, 115, 116 , and 117 iTRAQ reagents, respectively. Labeled peptides were then prefractionated off-line by SCX chromatography and analyzed by reverse-phase LC-MS. MS/MS spectra of iTRAQ-labeled peptides from Rab3a and Rab18 are illustrated. The peak areas of reporter ions 114, 115, 116, and 117 in the MS/MS spectra correspond to the relative abundance of the Rab proteins in the various brain homogenates. c, Quantitative proteomic analysis of Rabs in isolated rat brain homogenates. Data represent the means \pm SD of iTRAQ ratios from two biological replicates and are expressed as relative abundance over starting homogenate. Segmented line denotes enrichment threshold.

Rab1b, Rab2, Rab6, and Rab33b (Fig. 2b). These assignments corroborate their known affiliations on secretory vesicles, early and recycling endosomes, and ER/Golgi membranes in other cell systems.

\section{Rabs enriched on SVs localize to presynaptic boutons in primary hippocampal neurons}

The data described above lend support to the idea that purified SVs contain a subset of Rabs that are selectively enriched and thus may govern SV trafficking in the synapse. To confirm that these 
Table 1. Complete survey of the rat SV Rabome

\begin{tabular}{|c|c|c|c|c|c|c|c|c|c|c|c|c|}
\hline \multirow{2}{*}{$\begin{array}{l}\text { Rab } \\
\text { isoform }\end{array}$} & \multirow[b]{2}{*}{ Gi number } & \multirow{2}{*}{$\begin{array}{l}\text { Quantified } \\
\text { by }\end{array}$} & \multicolumn{3}{|c|}{ WB ratio ( $\pm S D$ ) } & \multicolumn{3}{|c|}{ iTRAQ ratio (土SD) } & \multicolumn{3}{|c|}{ Localization by } & \multirow{2}{*}{$\begin{array}{l}\text { GDI Pool } \\
\text { IP/MS detection }\end{array}$} \\
\hline & & & $\mathrm{S} 3 / \mathrm{H}$ & $\mathrm{LP} 2 / \mathrm{H}$ & $\mathrm{SV} / \mathrm{H}$ & $\mathrm{S} 3 / \mathrm{H}$ & $\mathrm{LP} 2 / \mathrm{H}$ & $\mathrm{SV} / \mathrm{H}$ & EGFP & $A B$ & SB labeling & \\
\hline Rab1a & gi| 45433570 & MS \& WB & $0.47(0.33)$ & $2.86(0.52)$ & $3.60(0.61)$ & $0.12(0.29)$ & $2.87(0.19)$ & $5.29(0.63)$ & Yes & Yes & No & Yes \\
\hline Rab2a & gi|13929006 & MS \& WB & $0.34(0.08)$ & $2.93(0.04)$ & $3.79(0.41)$ & $0.39(0.05)$ & $2.81(0.22)$ & $4.93(0.96)$ & $\mathrm{N} / \mathrm{A}$ & Yes & No & Yes \\
\hline Rab2b & gi|83415090 & MS & $0.34(0.08)$ & $2.93(0.04)$ & $3.79(0.41)$ & $0.36(\mathrm{~N} / \mathrm{A})$ & $1.04(\mathrm{~N} / \mathrm{A})$ & $1.06(\mathrm{~N} / \mathrm{A})$ & N/A & Yes & No & Yes \\
\hline Rab3a & gi|61098195 & MS \& WB & $2.43(0.32)$ & $7.66(0.16)$ & $12.42(0.72)$ & $0.38(0.04)$ & $6.11(1.52)$ & $13.07(1.40)$ & Yes & Yes & Strong & Yes \\
\hline Rab3b & gi| 13592037 & MS \& WB & $1.05(0.28)$ & $4.98(0.32)$ & $10.53(0.73)$ & $0.41(0.01)$ & $4.23(1.38)$ & $6.62(0.56)$ & $\mathrm{N} / \mathrm{A}$ & Yes & Strong & Yes \\
\hline Rab3c & gi||19424194 & MS \& WB & $0.45(0.02)$ & $6.30(0.82)$ & $9.89(1.85)$ & $0.30(0.01)$ & $6.80(0.31)$ & 19.04 (1.84) & Yes & N/A & Strong & Yes \\
\hline Rab4a & gi| 77404180 & WB & $1.30(0.03)$ & $1.80(0.89)$ & $5.01(0.24)$ & $\mathrm{N} / \mathrm{A}$ & N/A & $\mathrm{N} / \mathrm{A}$ & $\mathrm{N} / \mathrm{A}$ & $\mathrm{N} / \mathrm{A}$ & N/A & Yes \\
\hline Rab4b* & gi| 8394136 & MS \& WB & $1.30(0.03)$ & $1.80(0.89)$ & $5.01(0.24)$ & $0.05(\mathrm{~N} / \mathrm{A})$ & $0.90(\mathrm{~N} / \mathrm{A})$ & $1.84(\mathrm{~N} / \mathrm{A})$ & Yes & N/A & Weak & Yes \\
\hline Rab5a & gi|12083645 & MS \& WB & $1.24(0.03)$ & $2.63(0.60)$ & $6.91(0.27)$ & $0.04(0.25)$ & $2.18(0.19)$ & $5.30(1.35)$ & Yes & Yes & Moderate & Yes \\
\hline Rab5b & gi| 121583768 & MS \& WB & $1.24(0.53)$ & $2.63(0.60)$ & $6.91(0.27)$ & $0.35(0.18)$ & $2.79(0.70)$ & $5.68(0.20)$ & $\mathrm{N} / \mathrm{A}$ & Yes & Moderate & Yes \\
\hline Rab5c & gi| 157786692 & MS \& WB & $1.24(0.53)$ & $2.63(0.60)$ & $6.91(0.27)$ & $\mathrm{N} / \mathrm{A}$ & $1.51(\mathrm{~N} / \mathrm{A})$ & $3.30(\mathrm{~N} / \mathrm{A})$ & $\mathrm{N} / \mathrm{A}$ & Yes & Moderate & Yes \\
\hline Rab6a & gi|213972608 & MS \& WB & $1.54(0.36)$ & $1.35(0.48)$ & $1.68(0.42)$ & $0.73(\mathrm{~N} / \mathrm{A})$ & $2.58(\mathrm{~N} / \mathrm{A})$ & $2.59(\mathrm{~N} / \mathrm{A})$ & $\mathrm{N} / \mathrm{A}$ & Yes & No & Yes \\
\hline Rab6b & gi|157817539 & MS \& WB & $1.54(0.36)$ & $1.35(0.48)$ & $1.68(0.42)$ & $0.54(\mathrm{~N} / \mathrm{A})$ & $2.28(\mathrm{~N} / \mathrm{A})$ & $3.37(\mathrm{~N} / \mathrm{A})$ & Yes & Yes & No & Yes \\
\hline Rab7a & gi|13027392 & MS \& WB & $1.06(0.87)$ & $1.11(0.27)$ & $4.47(0.67)$ & $2.29(0.05)$ & $2.13(0.24)$ & $3.71(0.16)$ & Yes & N/A & No & Yes \\
\hline Rab8b & gi|23463313 & MS & N/A & N/A & $\mathrm{N} / \mathrm{A}$ & $\mathrm{N} / \mathrm{A}$ & $1.59(\mathrm{~N} / \mathrm{A})$ & $1.43(\mathrm{~N} / \mathrm{A})$ & Yes & N/A & No & Yes \\
\hline Rab10 & gi|61889071 & MS \& WB & $0.34(0.44)$ & $3.36(0.62)$ & $6.30(0.3)$ & $0.28(0.01)$ & $2.81(0.38)$ & $6.33(0.09)$ & Yes & $\mathrm{N} / \mathrm{A}$ & Moderate & Yes \\
\hline Rab11b & gi|14249144 & MS \& WB & $0.76(0.07)$ & $3.28(0.02)$ & $4.67(0.20)$ & $0.46(0.22)$ & $3.01(0.15)$ & $4.28(0.63)$ & Yes & N/A & Moderate & Yes \\
\hline Rab12* & gi| 158186685 & - & $\mathrm{N} / \mathrm{A}$ & N/A & $\mathrm{N} / \mathrm{A}$ & N/A & N/A & N/A & Yes & $\mathrm{N} / \mathrm{A}$ & No & Yes \\
\hline Rab14 & gi|16758368 & MS \& WB & $0.74(0.17)$ & $3.74(0.08)$ & $6.94(0.42)$ & $0.52(0.02)$ & $3.99(0.58)$ & $9.95(0.75)$ & Yes & $\mathrm{N} / \mathrm{A}$ & Moderate & Yes \\
\hline Rab15 & gi|38454238 & MS & $\mathrm{N} / \mathrm{A}$ & $\mathrm{N} / \mathrm{A}$ & $\mathrm{N} / \mathrm{A}$ & $\mathrm{N} / \mathrm{A}$ & $2.79(0.32)$ & $3.76(0.26)$ & N/A & $\mathrm{N} / \mathrm{A}$ & $\mathrm{N} / \mathrm{A}$ & No \\
\hline Rab18 & gi|60223069 & MS & N/A & N/A & N/A & $0.12(0.38)$ & $2.94(0.17)$ & $2.05(0.06)$ & Yes & $\mathrm{N} / \mathrm{A}$ & No & Yes \\
\hline Rab21 & gi|51948448 & MS & $\mathrm{N} / \mathrm{A}$ & $\mathrm{N} / \mathrm{A}$ & N/A & $\mathrm{N} / \mathrm{A}$ & $3.22(0.94)$ & $2.64(0.55)$ & $\mathrm{N} / \mathrm{A}$ & $\mathrm{N} / \mathrm{A}$ & $\mathrm{N} / \mathrm{A}$ & Yes \\
\hline Rab26* & gi| 19424272 & MS & $\mathrm{N} / \mathrm{A}$ & $\mathrm{N} / \mathrm{A}$ & $\mathrm{N} / \mathrm{A}$ & $\mathrm{N} / \mathrm{A}$ & N/A & $\mathrm{N} / \mathrm{A}$ & $\mathrm{N} / \mathrm{A}$ & $\mathrm{N} / \mathrm{A}$ & $\mathrm{N} / \mathrm{A}$ & Yes \\
\hline Rab27b & gi| 16758202 & WB & $0.22(0.29)$ & $4.03(0.65)$ & $9.95(0.31)$ & $\mathrm{N} / \mathrm{A}$ & N/A & N/A & Yes & Yes & Strong & No \\
\hline Rab33b & gi|157822117 & MS \& WB & $1.33(0.57)$ & $2.10(0.44)$ & $3.93(1.06)$ & $0.36(\mathrm{~N} / \mathrm{A})$ & $1.04(\mathrm{~N} / \mathrm{A})$ & $1.06(\mathrm{~N} / \mathrm{A})$ & Yes & N/A & No & Yes \\
\hline Rab34/39\# & gi|58865850/gi|157822741 & MS \& WB & 0.44 & $2.45(0.81)$ & $3.98(0.63)$ & $\mathrm{N} / \mathrm{A}$ & $\mathrm{N} / \mathrm{A}$ & $\mathrm{N} / \mathrm{A}$ & $\mathrm{N} / \mathrm{A}$ & $\mathrm{N} / \mathrm{A}$ & $\mathrm{N} / \mathrm{A}$ & No \\
\hline Rab35 & gi|61556789 & MS \& WB & $0.17(0.06)$ & $2.61(0.34)$ & $4.34(0.08)$ & $0.63(\mathrm{~N} / \mathrm{A})$ & $1.85(\mathrm{~N} / \mathrm{A})$ & $1.96(\mathrm{~N} / \mathrm{A})$ & Yes & Yes & No & Yes \\
\hline Rab43 & gi|66730539 & - & $\mathrm{N} / \mathrm{A}$ & N/A & $\mathrm{N} / \mathrm{A}$ & N/A & $\mathrm{N} / \mathrm{A}$ & N/A & N/A & $\mathrm{N} / \mathrm{A}$ & N/A & No \\
\hline
\end{tabular}

*Peptide redundancy. Rab4b: 1a, 3a-c, 4a, 4b, 8b, 10, 12; Rab12: 1a, 3a-b, 4a, 8a, 10, 14, 35; Rab26: 1a, 3a-b, 4a, 8a, 10, 14, 35.

\#Unique peptide poor quality for quantitation. Rab34/39.

Note that localization studies were performed using EGFP-fused Rabs and/or Rab-specific antibodies (AB). SB labeling was scored weak-to-strong based on Rab colocalization and correlation with synaptophysin immunostaining. N/A, Not applicable; WB, Western blotting.

Rabs are indeed specifically associated with presynaptic nerve terminals, we next transiently expressed each Rab as an enhanced green fluorescent protein (EGFP) fusion chimera in primary rat hippocampal neurons (10-12 DIV) and analyzed by fluorescence microscopy which of these localized to synaptic boutons (SBs). GFP chimeras are routinely used to study the localization and function of Rab proteins, including those investigated here, and despite concerns about possible mistargeting, they have been shown in many cases to localize identically to their endogenous counterparts (de Hoop et al., 1994; Tiwari et al., 2003; Star et al., 2005; Tsuboi and Fukuda, 2006; Handley et al., 2007; Dejgaard et al., 2008). To aid the visualization of SBs and dendrites, we costained all transfected neurons with antibodies against synaptophysin (Syph, red) and MAP2 (blue), respectively. A full survey of the subcellular localization patterns of 16 candidate SV Rabs is presented Table 1 and supplemental Figure 2 (available at www. jneurosci.org as supplemental material).

Expression in rat hippocampal neurons revealed that seven of these Rab fusion chimeras fully or partially colocalized with synaptophysin in synaptic nerve terminals (Fig. 3, yellow overlap). Consistent with their strong biochemical SV enrichment, Rab3a and Rab27b were exclusively localized to axonal SBs where SVs are accumulated. Rab4b, Rab5a, Rab10, Rab11b, and Rab14 also showed EGFP labeling on select SBs (Fig. 3). Additionally, a subset of these GTPases displayed prominent EGFP targeting on perinuclear puncta reminiscent of endosomal and/or Golgi membranes (supplemental Fig. 3, available at www.jneurosci.org as supplemental material). In some instances, we also observed labeling of dendrites/dendritic spines in more mature neurons, particularly upon more pronounced protein overexpression
(N. J. Pavlos, unpublished observations). By comparison, Rab1b and Rab2a were largely confined to the Golgi in neuronal cell bodies as confirmed by immunostaining with the cis-Golgi marker GM130 (supplemental Fig. 4, available at www.jneurosci. org as supplemental material). Where possible, we compared the subcellular distribution patterns of EGFP-tagged Rabs with that of the endogenous counterparts using immunocytochemistry of nontransfected cells, resulting in very similar staining patterns (Table 1; supplemental Fig. 4, available at www.jneurosci.org as supplemental material).

Cross-correlation and quantitative immunofluorescence analyses of individual axons (Fig. 4a) and SBs (Fig. 4b) further supported these localizations, with Rab27b, Rab11, and Rab14 demonstrating strong correlation with Rab3a and Syph double-labeled SBs; Rab4b, Rab5a, and Rab10 showing modest correlation; and little to no correlation being observed among several other indicated Rabs or EGFP alone, which served a negative control. Overall, among the 16 EGFP-Rabs examined, 7 colocalized, at least partially, with SBs in good accord with the results of the proteomic and immunoblot analyses.

\section{Rab3a and Rab27b reside on distinct yet overlapping SV pools} Rab27 proteins (Rab27a/b) have recently emerged as key regulators of exocytotic membrane trafficking sharing several intrinsic characteristics with Rab3 family members, including the following: (1) sequence homology; (2) localization on secretory organelles; and (3) divalent regulators (Rab3GEF) and effectors (e.g., RIM and Rabphilin) (Fukuda, 2008). Moreover, like Rab3s, Rab27 proteins have also been implicated in synaptic transmis- 
sion in invertebrates (Mahoney et al., 2006; Yu et al., 2008); but their precise subcellular localization has been unclear.

In the following experiments, we have compared the subcellular localization of Rab27b with that of Rab3a using complementary approaches to determine whether they reside on identical or different synaptic vesicle pools. First, we compared the subcellular distribution patterns of Rab27b and Rab3a with synaptophysin in cultured hippocampal neurons by triplelabeled immunofluorescence microscopy (Fig. 5a). For this purpose, rat hippocampal neurons grown on glass coverslips were cultured to 17 DIV followed by immunolabeling using antibodies specific to Rab3a, Rab27b, and Syph, respectively. Consistent with their GFP protein targeting in fixed (Fig. 3) and live neurons (supplemental Fig. 5, Video 1, available at www.jneurosci.org as supplemental material), Rab3a and Rab27b strongly colocalized with synaptophysin in SBs (Fig. $5 a)$. Closer examination of synaptic nerve terminals by ultrathin sectioning and confocal microscopy unveiled notable differences between Rab27b- and Rab3a-labeled puncta (Fig. 5b, arrows). Immunogold EM of purified SVs revealed that both Rab3a and Rab27b are present on SV membranes, with gold particles detectable on the surface of most, but not all, SVs (Fig. 5c).

Next, we compared the sedimentation profiles of Rab3a and Rab27b in subfractionated synaptosomes by density centrifugation (Fig. $5 d$ ). For this, osmotically lysed synaptosomes were overlaid on a 0.4-1.2 M continuous sucrose gradient and subjected to high-speed centrifugation $(100,000 \times g)$. The resulting gradient was fractionated from the bottom, and equal volumes of gradient fractions were analyzed by SDS-PAGE and immunoblotting using antibodies against the indicated proteins. As expected, Rab3a migrated in cytosolic (Cyt; F:1), SV (F:2-5), and plasma membrane (PM; F:23-26) enriched fractions as evidenced by its cosedimentation with marker proteins RabGDI, synaptophysin, and $\mathrm{Na}^{+} / \mathrm{K}^{+}$-ATPase, respectively. Rab27b immunoreactivity also coincided with Rab3a in both SV and PM fractions (the latter containing docked SVs). However, in contrast to Rab3a, little Rab27b signal was observed in the cytosolic fraction.

The differential localization of Rab3a and Rab27 described above (Fig. $5 b$ ) prompted us to investigate whether Rab3a and Rab27b occupy the same or at least partially distinct pools of synaptic vesicles. To address this question, we immunoisolated SVs using Immunobeads containing covalently coupled monoclonal antibodies specific for Rab3a (Fischer von Mollard et al., 1994b). The capture efficiency, uniformity, and specificity of the Rab3a-isolated SVs was confirmed by EM morphometry (Fig. 5e) and immunoblotting (Fig. 5f). Vesicles isolated with the Rab3aImmunobeads contained Rab27b, but not Rab6 (Golgi Rab), b
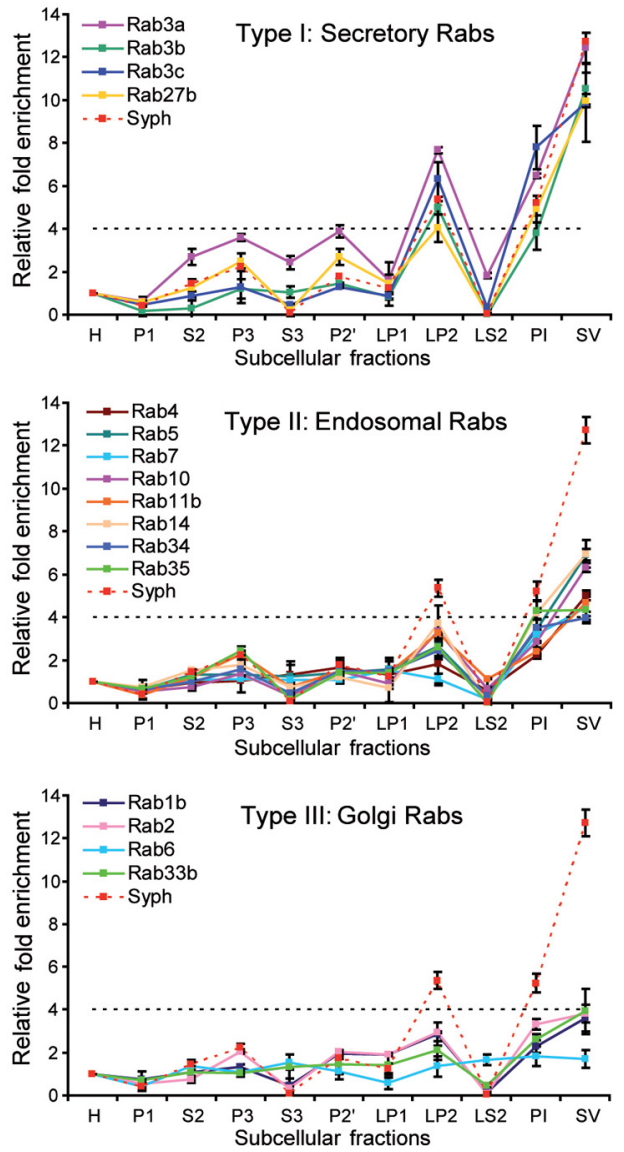

I

Enrichment profiles of Rab GTPases on purified SVs isolated from rat brain homogenates, determined by quantitative SV by differential centrifugation and controlled pore glass chromatography. Five micrograms of each fraction were resolved by ( \pm SD) over homogenate of three independent blots. Segmented lines correspond to the synaptophysin enrichment profile (red) and threshold (black), respectively.

which was used as control. Comparison of the enrichments of Rab3a and Rab27b revealed that binding of Rab27b-containing organelles was only approximately half $(\sim 45 \%)$ as efficient as that of Rab3-containing organelles (Fig. $5 g$ ), suggesting that Rab27b occupies distinct yet intersecting SV pools with Rab3a. While we cannot exclude that the unbound population of Rab27b-containing organelles also includes non-SV membranes, these data collectively (1) affirm the residency of Rab27b as a bona fide SV constituent and (2) suggest that Rab3a and Rab27b localize to partially distinct pools of SVs.

Rab3a and Rab27b exhibit differential dynamics on SV membranes during $\mathrm{Ca}^{2+}$-triggered exocytosis

Rab3s have been previously shown to dissociate from SV membranes upon stimulation of exocytosis (Fischer von Mollard et al., 1991, 1994a; Star et al., 2005). Dissociation is reversible and is associated with a shift from the GTP to the GDP form. To assess whether Rab27b exhibits a similar SV cycling pattern, isolated rat nerve terminals (synaptosomes) were depolarized by $\mathrm{K}^{+}$addition (Fig. $6 a$, arrow), resulting in a robust, $\mathrm{Ca}^{2+}$-dependent release of glutamate. Following exocytosis, the same synapto- 

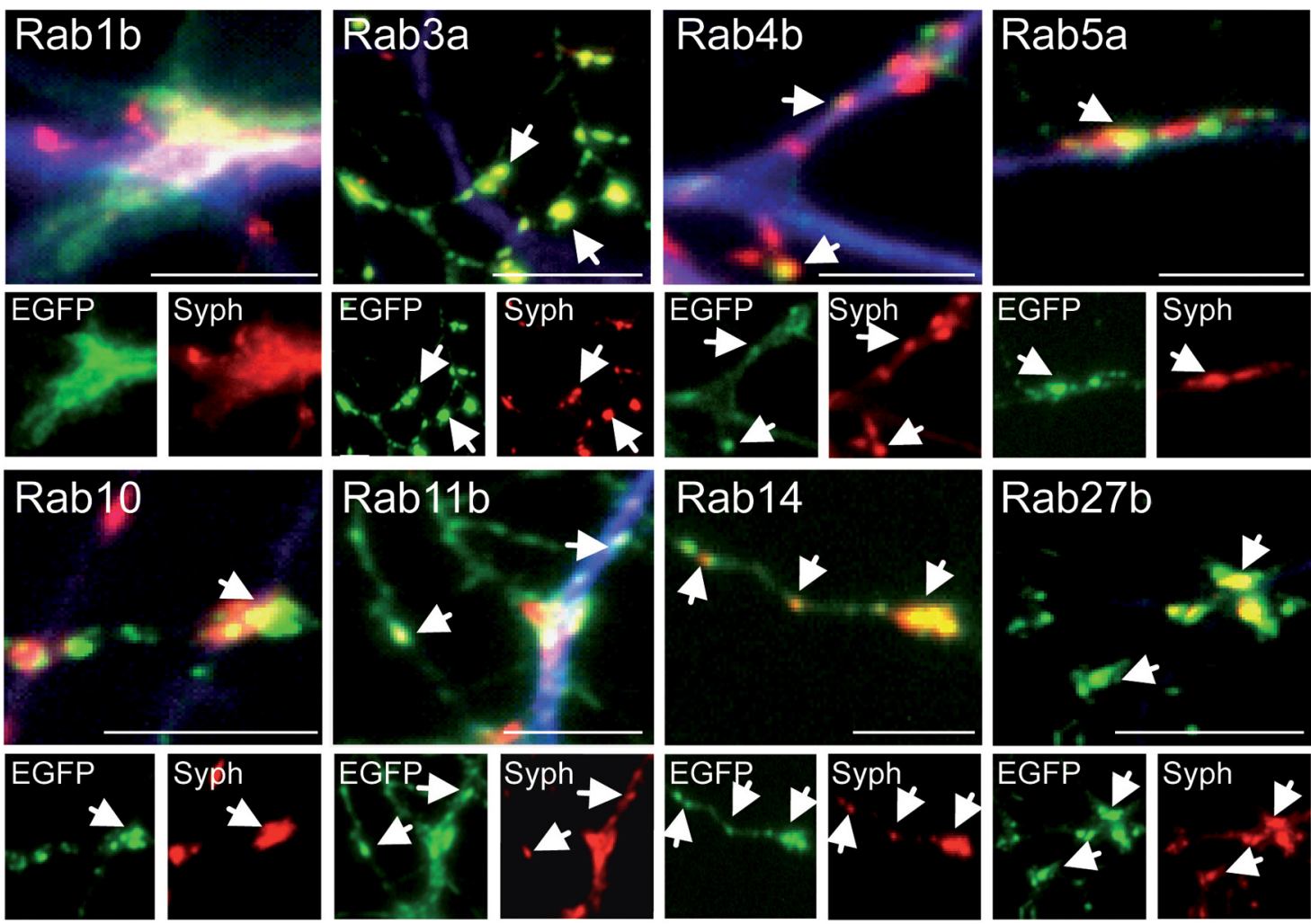

Figure 3. A survey of the subcellular localization of SV-enriched Rabs in rat hippocampal neurons. pEGFP-C2-Rab3a,-Rab4b,-Rab5a,-Rab10,-Rab11b,-Rab14, and-Rab27b were each transiently transfected into primary rat hippocampal neurons (10-12 DIV). Forty-eight hours after transfection, neurons were fixed and immunostained with antibodies against Syph (red) and MAP2 (blue) to visualize synaptic boutons and dendrites, respectively. Areas of overlap appear yellow. Arrows highlight colabeled structures. Scale bar, $20 \mu \mathrm{m}$.

somes were rapidly pelleted and osmotically lysed, and the released SVs were harvested by high-speed centrifugation. Immunoblot analysis of the SVs revealed that, as expected, both Rab3a and Rab3c dissociated from SV membranes following stimulation of exocytosis (Fig. 6b,c). By comparison, no significant change was observed in the levels of SV-bound Rab27b and Rab5 after stimulation (Fig. 6b). Differential Rab3a/Rab27b membrane dissociation was also observed at the confocal microscopic level using ultrathin sections of stimulated SBs from rat hippocampal neurons (Fig. 6d,e). In this instance, Rab3a immunofluorescence was reduced following $\mathrm{K}^{+}$depolarization (50 $\mathrm{mM}, 1 \mathrm{~min}$ ), whereas Rab27b signals remained steady under stimulated and control conditions.

$\mathrm{Ca}^{2+}$-triggered dissociation of Rab3 proteins from SVs is thought to reflect conversion of GTP-Rab3 to GDP-Rab3, followed by removal of GDP-Rab3 from the membrane by Rab-GDI (Chou and Jahn, 2000). Thus, it is conceivable that stimulation of neurotransmitter release does not cause GTP cleavage by Rab27b, in contrast to Rab3a. Alternatively, it is possible that GDI is unable to extract even the GDP form of Rab27, which may explain the unusually low levels of Rab27b in the soluble cytoplasm. To test this, we preincubated purified SVs with either GDP or GTP $\gamma$ S $(500 \mu \mathrm{M})$ and then added purified recombinant GDI (200 nM) for $45 \mathrm{~min}$, after which SVs were pelleted and analyzed for the presence of select Rab proteins (Fig. 6f). As expected, Rab3a, Rab5, and Rab6 were effectively extracted from SVs by GDI when incubated in the presence of excess GDP, but not in the presence of the nonhydrolyzable analog GTP $\gamma \mathrm{S}$. In contrast, Rab27b was resistant to GDI-induced membrane extraction, even at high GDI concentrations (up to $2 \mu \mathrm{M}$ ). Notably, addition of GDI resulted in a peculiar Rab27b doublet (arrows) perhaps indicative of a posttranslational modification or cross-reactivity with a contaminating peptide. This doublet was not observed for any other Rabs examined, including Rab18, which has been similarly shown to be GDI resistant (Liu et al., 2007). We also isolated GDI-Rab complexes from the presynaptic cytosol using affinity chromatography with a GDI-specific monoclonal antibody and analyzed GDI-bound Rabs (Fig. 6g). While this approach captured the bulk of Rabs uncovered in our original MS screen, we were unable to detect Rab27b in the presynaptic GDI-bound Rab pool (Fig. 6h). These data show that Rab27b, unlike Rab3a, is resistant to extraction by GDI, resulting in a different membrane dissociation dynamics during $\mathrm{Ca}^{2+}$-triggered neurotransmitter release.

\section{Rab27b regulates SV recycling in hippocampal neurons}

The data described so far establish Rab27b as a bona fide resident of SVs, but it remains unclear whether the protein exerts a function in SV exocytosis and/or recycling. To address this issue, we next performed SV recycling assays on hippocampal neurons transiently transfected with either wild-type (wt) EGFP-Rab27b or mutant variants that interfere with GTP hydrolysis (Q78L: GTP-locked) and/or GDP/GTP exchange (T23N: nucleotide-reduced; N133I: nucleotide-empty), respectively (Tiwari et al., 2003). Expression in rat hippocampal neurons (Fig. 7a,b) showed similar localizations for EGFP-Rab27b ${ }^{\text {WT }}$ and -Rab27b ${ }^{\text {Q78L }}$ proteins that were characteristic of SBs as revealed by fluorescence microscopy. In comparison, EGFP-Rab27b ${ }^{\text {T23N }}$ and -Rab27b ${ }^{\mathrm{N} 133 \mathrm{I}}$ displayed diffuse distribution patterns, largely concentrated in the soma of neurons with little to no specific SB labeling observed. Overexpression of these mutant chimeras did not reveal any obvious disruptions in the morphology, number, and/or positioning of synaptophysin-labeled SBs or dendrite outgrowth 

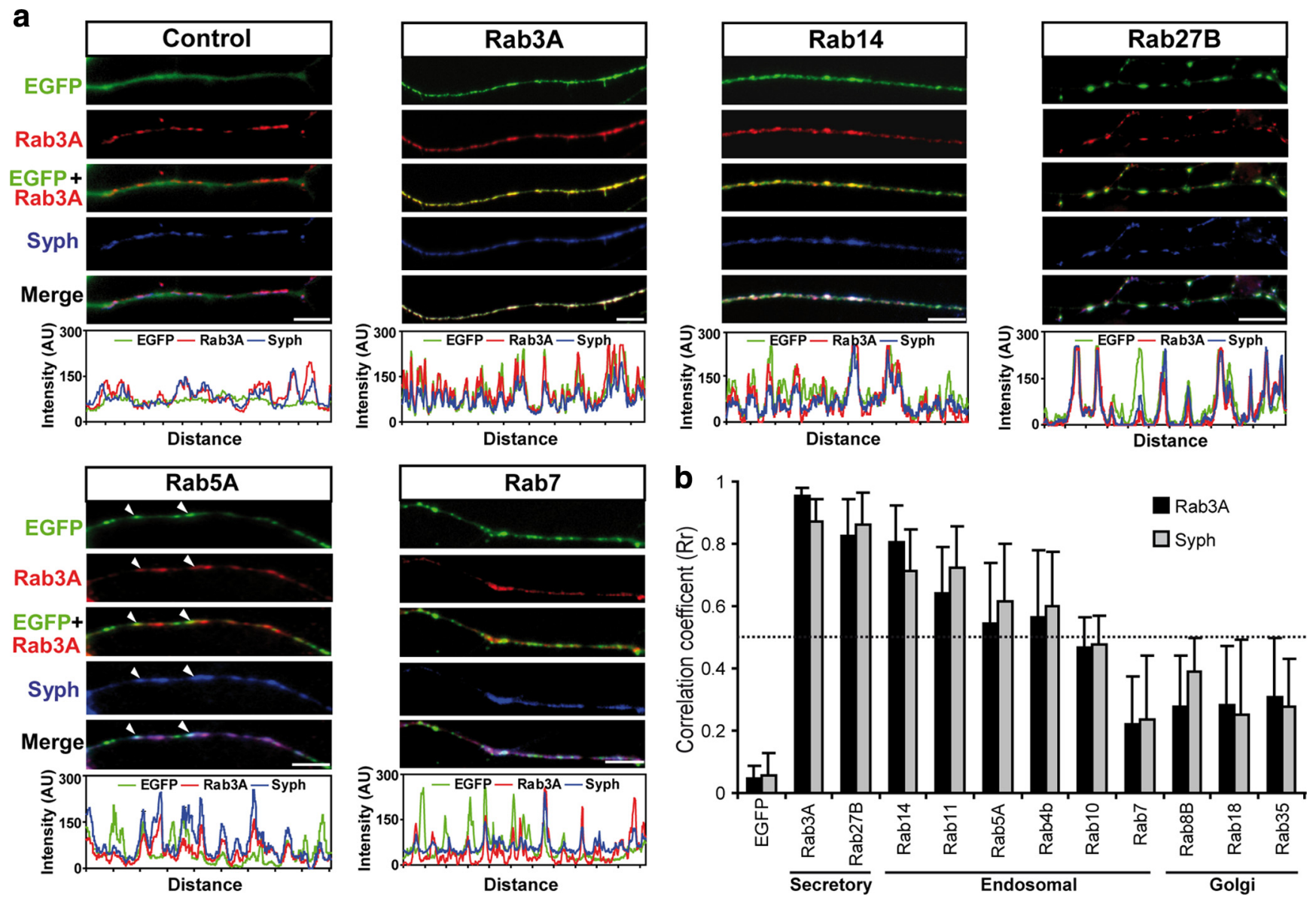

Figure 4. Quantitative immunofluorescence analysis of SV Rabs in rat hippocampal nerve terminals. $\boldsymbol{a}$, Representative axons of hippocampal neurons (10-12 DIV) transfected with the indicated EGFP-Rab construct and fixed $48 \mathrm{~h}$ after transfection. Neurons were subsequently double labeled with antibodies against Rab3a and Syph to visualize synaptic boutons, and correlative line scans were performed across the entire length of the axons with corresponding histograms illustrated below. Scale bar, $5 \mu \mathrm{m}$. $\boldsymbol{b}$, Cross-correlation analyses of EGFP-Rabs with either Rab3a (black bars) or synaptophysin (gray bars) on individual synaptic boutons $(n=50)$. Secretory Rabs $\operatorname{Rab3a}(R r=0.956)$ and $\operatorname{Rab27b}(R r=0.828)$ showed strong correlation with the respective SV markers, whereas $\operatorname{Rab35}(R r=0.310)$ showed no obvious correlation. Data represents the means \pm SD.

(supplemental Fig. 6, available at www.jneurosci.org as supplemental material); however, we did observe notable reductions in the endogenous immunofluorescence levels of Rab3a upon high expression of the Rab27b ${ }^{\text {T23N }}$ and Rab27b ${ }^{\text {N133I }}$ (more prominent) mutants (Fig. 7c). Possibly, this reflects reduced activation of Rab3a due to competition for a common Rab3/Rab27-GEF (Figueiredo et al., 2008), which dominant-negative Rab mutants are known to bind with high affinity (Burstein et al., 1992; Chen et al., 2003).

SV recycling was assessed by measuring the uptake of the fixable membrane dye FM4-64FX in presence of controlled stimulation using an electric field stimulator. For this purpose, rat hippocampal neurons transiently expressing EGFP-Rab27b ${ }^{\text {WT }}$ and mutant proteins were labeled with FM4-64FX by bath application and then electrically stimulated (600 APs, $20 \mathrm{~Hz})$. The neurons were then fixed, and FM4-64FX uptake monitored by confocal microscopy (Fig. 7d). When comparing FM4-64FX uptake in axons of transfected neurons with their nontransfected neighbors, we found no obvious difference in axonal SB fluorescence intensity upon Rab27b ${ }^{\text {WT }}$ overexpression. On the other hand, averaging the amount of FM4-64FX uptake in hundreds of SBs from multiple cells/fields revealed strong reductions in FM4-64FX dye uptake in all Rab27b mutants with a relatively potency of Rab27b ${ }^{\text {N133I }}>$ Rab27b ${ }^{\text {Q78L }}>$ Rab27b $^{\text {T23N }}$ (Fig.
$7 d, e)$. Together, these data posit a role for Rab27b in neuronal SV recycling.

\section{Discussion}

In the present study, we have combined highly purified organelle fractions with state-of-the-art high-resolution quantitative proteomics, immunoanalytical validation, and fluorescence microscopy to clarify which Rab proteins are specifically associated with the membrane of SVs. Based on our analyses, we conclude that SVs contain a set of Rabs functioning both in exocytosis and endosomal recycling, including Rab3a, Rab3b, Rab3c, Rab4b, Rab5a, Rab10, Rab11b, Rab14, and Rab27b. Furthermore, we show that Rab27b is involved in the regulation of SV exocytosis and exhibits both overlapping and distinct features with Rab3 proteins, suggesting that these Rabs act in concert to endow SV exocytosis with both flexible and robust features.

\section{The SV Rabome}

Rab proteins are generally considered to be the key determinants of organellar identity and organellar subdomains in the secretory pathway. Thus, it is conceivable that the set of membrane-bound Rabs in the GTP form not only define the nature of the organelle but also orchestrate the subsequent trafficking step. As a first step toward identifying such organelle-specific sets, several Rabomes 
a
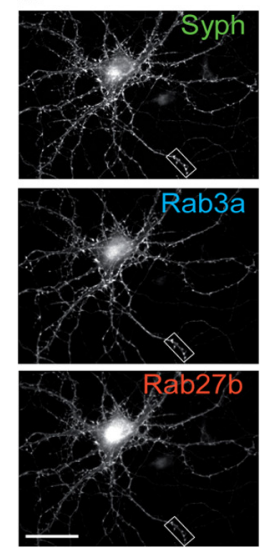

b
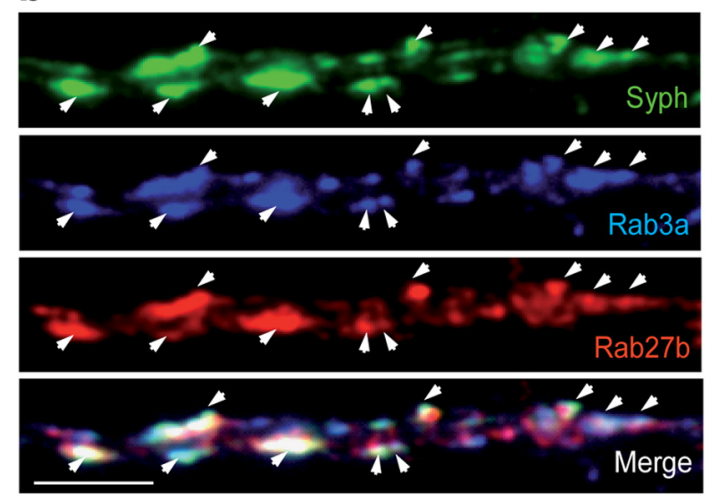

d
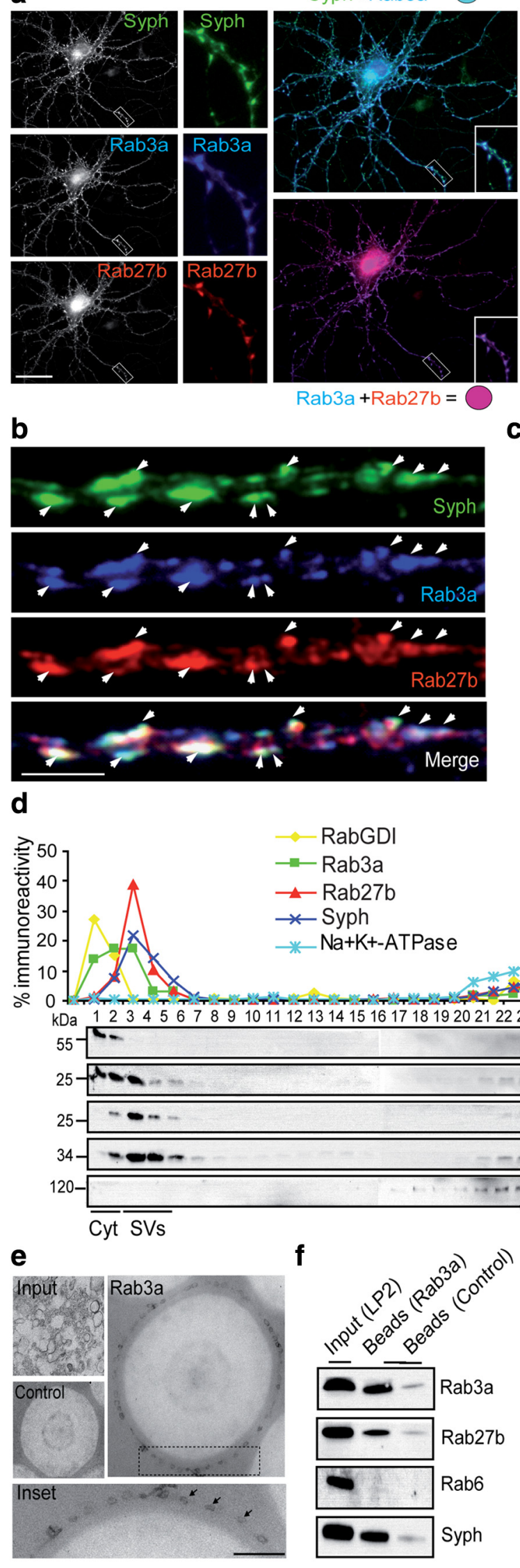

Rab3a + Rab27b $=$
Syph + Rab27b $=$
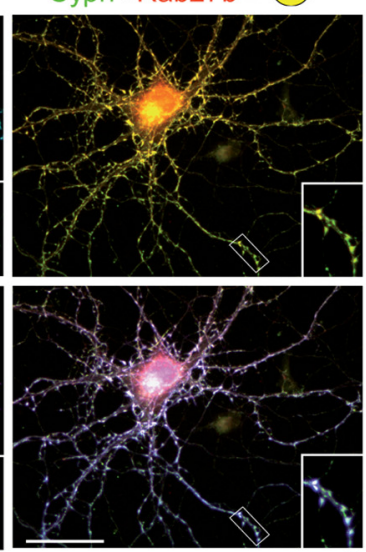

Syph +Rab3a+Rab27b =

C
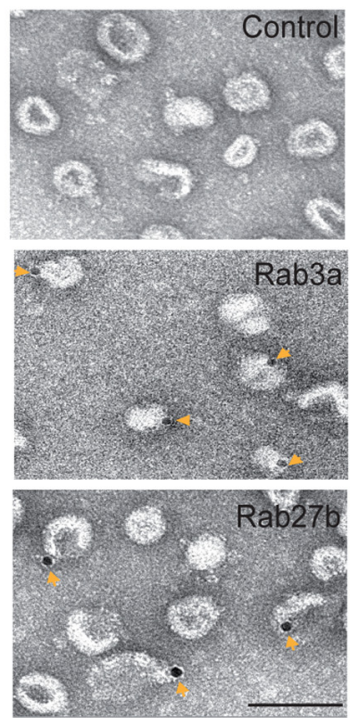
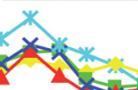

2425262728

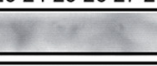

RabGD

7000

Rab3a

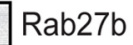

Syph $\mathrm{Na}+\mathrm{K}+-\mathrm{ATPase}$

PM

g

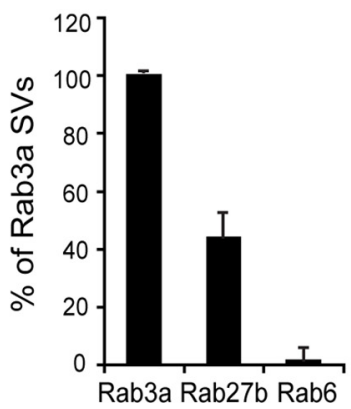

Figure 5. Rab27b occupies distinct but overlapping SV pools with Rab3a. a, Immunofluorescence analysis of Rab27b-, Rab3a-, and synaptophysin-labeled synaptic boutons. Hippocampal neurons (17 DIV) were fixed in 3\% PFA and triple labeled with

have been recently described using similar proteomic and image-based screens (Gilchrist et al., 2006; Takamori et al., 2006; Liu et al., 2007; Dejgaard et al., 2008). However, while these studies clearly define the bulk of membrane residing Rabs, they do not provide quantitative information concerning which of these GTPases are specifically enriched and which is not and thus is likely to be a contaminant. The multiple quantitative lines used in the present study have enabled us to accurately gauge, for the first time, the relative abundance of Rab proteins on an intracellular organelle. Although such an approach cannot guarantee the completeness the dataset obtained, particularly in light of the inherent protein redundancy that exists between closely related Rabs (as observed here with Rab3s and Rab27b), it is likely that the Rabs quantified and presented herein constitute the major SV cache.

Among the exo-endocytic Rabs enriched on SVs, members of the Rab3 (Rab3a/b/c) family were most prominent. This finding was not unexpected and reaffirms the wealth of published literature that have posited Rab3s as core components of the SV exocytotic machinery

$\leftarrow$

antibodies against Rab27b, Rab3a, and Syph. Insets represent magnifications of boxed regions. Colocalization appears white following the overlay of all three fluorescent channels. Scale bar, $10 \mu \mathrm{m} . \boldsymbol{b}$, Confocal micrograph of an ultrathin section of a neuronal axon triple labeled with Rab3a, Rab27b, and Syph antibodies. Fluorescently labeled neurons grown on coverslips were postfixed with $2 \%$ glutaraldehyde and embedded in 2,4,6-tris[bis(methoxymethyl)amino]-1,3,5-triazine, and ultrathin sections ( $\sim 100 \mathrm{~nm}$ ) were cut before sections were mounted for analysis by confocal microscopy. Arrows denote colocalized puncta. Note that the colocalization between Rab27b and Rab3a is incomplete in the merged panel. c, Immunogold localization of Rab3a and Rab27b on highly purified SVs. SV samples were fixed and incubated in the presence of either rabbit IgG (control), or antibodies against Rab3a or Rab27b. The samples were then incubated with appropriate secondary antibodies conjugated to $10 \mathrm{~nm}$ gold. Gold particles (yellow arrows) are distributed on the surface of select SVs. Scale bar, $200 \mathrm{~nm}$. $\boldsymbol{d}$, Velocity density gradient centrifugation analyses of subfractionated synaptosomes. Gradient fractions (0.4-1.2 м sucrose) were analyzed from top to bottom by quantitative immunoblotting of membranes for the SV marker Syph, Rab3a, Rab27b, cytosolic (Cyto) GDI, and the PM marker $\mathrm{Na}^{+} / \mathrm{K}^{+}$-ATPase. $\boldsymbol{e}$, Presence of Rab27b on SVs immunoisolated with Rab3a. SVs (LP2) were incubated with Eupergit C1Z beads containing covalently attached monoclonal antibodies to Rab3a (Cl 42.2) or incubated with beads whose functional groups had been inactivated with glycine (control). SV binding was monitored by EM morphometry. Inset denotes magnification of boxed region. Scale bar, $500 \mathrm{~nm}$. The co-occupancy of Rabs on Rab3a-immunoisolated SVs was analyzed by immunoblotting $(\boldsymbol{f})$, quantified $(\boldsymbol{g})$, and normalized to the total captured Rab3a SV pool. Means \pm SEM from three to five independent measurements are shown. 

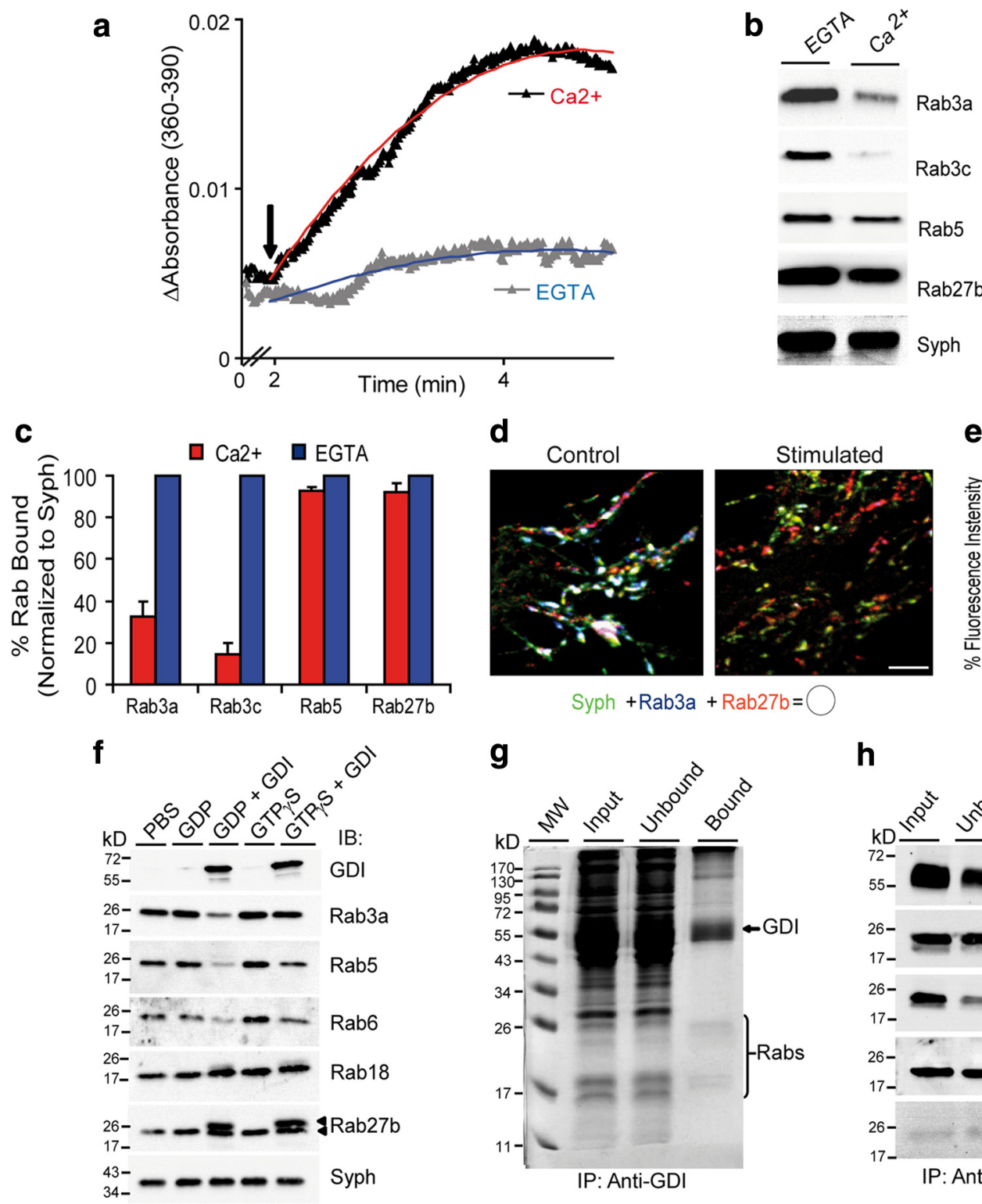

d

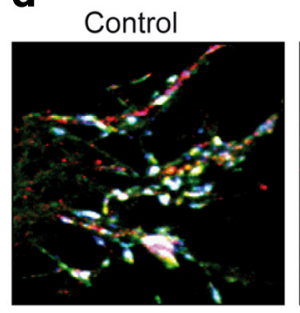

Syph +Rab3a +Rab27b= e

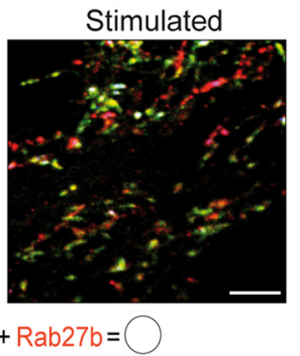

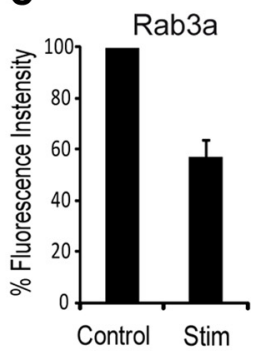

g

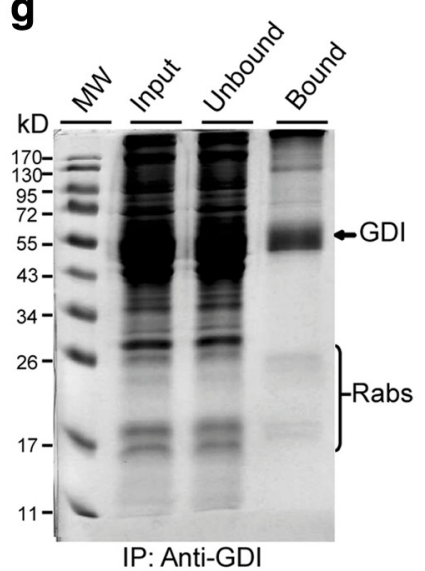

h

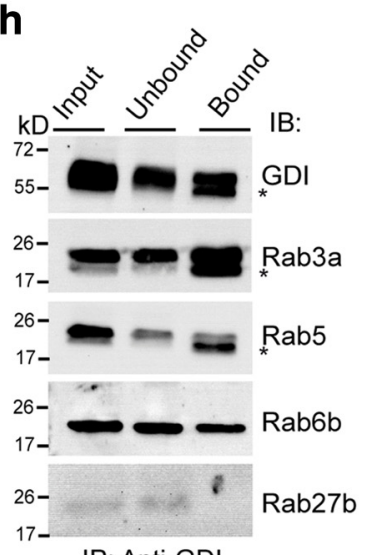

IP: Anti-GDI

Figure 6. Differential membrane dynamics between Rab27b and Rab3a during $\mathrm{Ca}^{2+}$-triggered exocytosis. $\boldsymbol{a}$, $\mathrm{Ca}^{2+}$-dependent stimulation of glutamate release by $\mathrm{K}^{+}$depolarization from isolated rat synaptosomes. Synaptosomes $(1 \mathrm{mg}$ ) were resuspended in sodium buffer containing $1.3 \mathrm{~mm}$ calcium chloride, 5 mm EGTA, or 50 mm KCl and glutamate release was monitored spectrophotometrically following the generation of NADPH associated with the conversion of glutamate dehydrogenase. $\boldsymbol{b}$, Dissociation of Rab3a/c but not Rab5 or Rab27b during Ca ${ }^{2+}$-triggered exocytosis. Following stimulation and release, synaptosomes were subfractionated to obtain crude SV factions (total assay duration $\sim 1 \mathrm{~h}$ ), which were analyzed by SDS-PAGE and immunoblotting with the indicated antibodies. Syph served as a loading control for equivalent amounts of SVs. C, Densitometric analyses of Rabs bound to SVs following Ca ${ }^{2+}$-triggered exocytosis. Ca ${ }^{2+}$ and EGTA immunointensities were normalized to their respective Syph controls and expressed as a percentage of the respective EGTA controls. $\boldsymbol{d}$, Dispersion of Rab3a but not Rab27b upon $\mathrm{K}^{+}$depolarization. Hippocampal neurons were incubated with neuronal buffer in either the presence (stimulated) or absence (control) of $\mathrm{KCl}$ ( $50 \mathrm{~mm}$ ) for $1 \mathrm{~min}$, immediately fixed, triple labeled with the indicated antibodies and processed for ultrathin sectioning of synaptic boutons for confocal microscopy. Representative confocal micrographs are depicted. White color denotes Rab3a/Rab27b/Syph colocalization following overlay of individual channels. $e$, Quantification of the relative Rab3a immunofluorescence intensity in the absence or presence of stimulation (stim). Data are expressed as a percentage of the no stimulation control.f, Rab27b is resistant to GDI-membrane extraction. Isolated SVs (LP2) were incubated for 15 min in the presence of GDP or GTP $\gamma S$ (500 $\mu \mathrm{m}$ ) at $37^{\circ} \mathrm{C}$. His-GDI ( $200 \mathrm{~nm}$ ) or PBS (control) was then added and incubation was continued for an additional $45 \mathrm{~min}$. The membranes were pelleted and then analyzed by SDS-PAGE and immunoblotting. In addition to Rab3a, GDI efficiently removes Rab5 and Rab6 from SV membranes but not Rab18 or Rab27b. Note that a distinct doublet band (arrows) is observed in the Rab27b samples following GDI incubation in both the presence of either GDP or GTP $\gamma$ S. $g$, Isolation of Rab-GDI complexes by affinity chromatography. Rab-GDI complexes from concentrated presynaptic cytosol (LS2) were captured by an affinity column coupled with a monoclonal antibody specific for GDI. Complexes were eluted by low pH and samples analyzed by immunoblotting (h) and mass spectrometry (see Table 1 ). Asterisks in $\boldsymbol{g}$ correspond to the light chain of the monoclonal antibody used for affinity purification. Note the low abundance of Rab27b in the starting input and flow through samples and low capture affinity with GDI as compared to other Rabs examined.

(Lang and Jahn, 2008). In fact, our previous analyses indicate that Rab3a alone accounts for $>2.5 \%$ of all known SV proteins $(\sim 10$ copies/vesicle) (Takamori et al., 2006). Closely imitating the expression and subcellular distribution patterns of Rab3 proteins, Rab27 GTPases (Rab27a/b) are enriched in a broad range of specialized tissues and cells types with high exocytic requirements where they localize to a variety of secretory vesicles (Fukuda, 2008). Unlike Rab27a, which is primarily expressed outside the CNS, the Rab27b isoform has been shown to be highly enriched in the brain (Zhao et al., 2002). More recently, Rab27a and Rab27b have been shown to play distinct and nonredundant roles on several secretory organelles (Johnson et al., 2010; Ostrowski et 
a
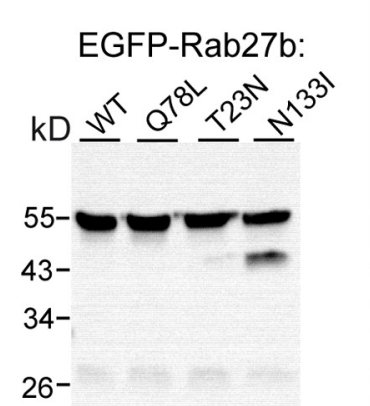

IB: Anti -GFP

C
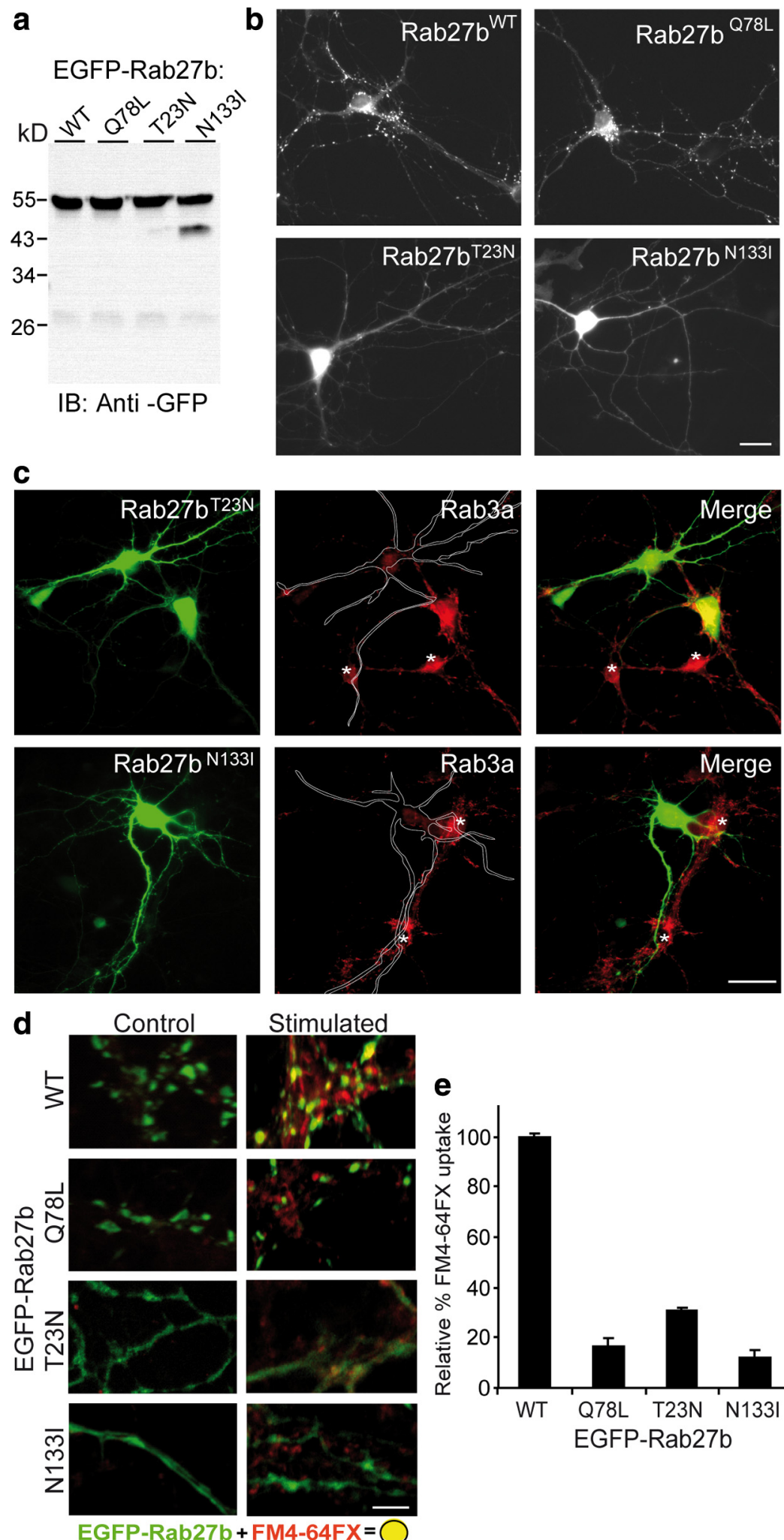

Stimulated
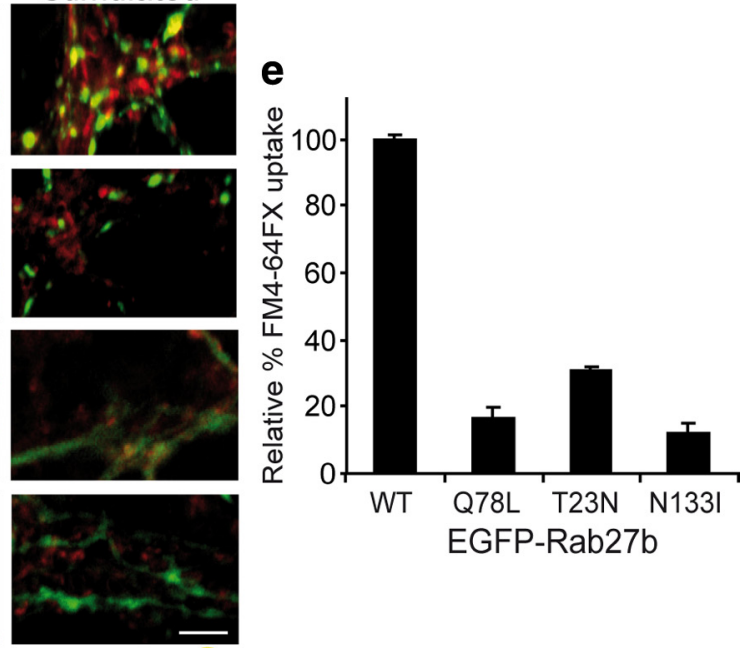

Figure 7. Functional characterization of Rab27b GTP/GDP-mutants in hippocampal neurons. $\boldsymbol{a}, \boldsymbol{b}$, Expression and localization of EGFP-Rab27b fusion proteins in rat hippocampal neurons. Hippocampal neurons (8 DIV) were transfected with the indicated EGFP-Rab27b fusion constructs for $48 \mathrm{~h}$ and then either harvested for analysis by SDS-PAGE and immunoblotting with an antibody against GFP $(\boldsymbol{a})$ or fixed and processed for fluorescence microscopy $(\boldsymbol{b})$. EGFP-Rab27b ${ }^{\text {WT }}$ and -Rab27b ${ }^{\text {Q78L }}$ mutants localized primarily to synaptic boutons, whereas EGFP-Rab27b ${ }^{T 23 \mathrm{~N}}$ and -Rab27b ${ }^{\mathrm{N} 1331}$ were diffusely distributed throughout the neuronal somas and radiating neurites. c, Overexpression of GDP-Rab27b mutants reduces membrane-bound Rab3a. Hippocampal neurons strongly expressing EGFP-Rab27b ${ }^{\mathrm{T} 23 \mathrm{~N}}$ and -Rab27b ${ }^{\mathrm{N} 1331}$ were immunostained with antibodies against endogenous Rab3a and examined by fluorescence microscopy. Overexpression of each EGFP-Rab27b fusion protein correlated with a reduction in Rab3a immunofluorescence levels as compared to untransfected neighboring cells (asterisks). Segmented line outlines boundaries of al., 2010). Our striking observation that Rab27b, but not Rab27a, is selectively and highly expressed on purified SVs further supports these divergent Rab27a/b localizations and functions and extends the organelle repertoire of Rab27b to SVs.

Along with the classical exocytotic components, several endocytic Rabs (Rab4b, Rab5a/b, Rab10, Rab11b, and Rab14) were also enriched on SVs, albeit to a lesser degree. As endocytic recycling at neurochemical synapses is characteristic of all SVs, it is not surprising that multiple endosomal species exist on SV membranes. For instance, the early endosomal Rab5 GTPase may facilitate SV targeting or tethering to endosomal intermediate compartments following clathrin-dependent endocytosis and SV uncoating. Indeed, Rab5 has been previously detected on SV membranes and implicated in SV recycling in synaptic nerve terminals (de Hoop et al., 1994; Fischer von Mollard et al., 1994b; Wucherpfennig et al., 2003; Star et al., 2005). Moreover, disruption of the Rab5 GTPase cycle correlates with morphological disturbances in SV-recycling intermediates and SV pools in both flies and rats (de Hoop et al., 1994; Shimizu et al., 2003; Star et al., 2005). Similar functions might also be envisaged for other endosomal SV candidates, including Rab4, Rab10, Rab11, and Rab14, which have been shown to cycle between endosomal and clathrin-coated micro-domains (Ullrich et al., 1996; Junutula et al., 2004). A tempting scenario presents a coordinated Rab recruitment cascade to newly recycled SVs and endosomal intermediates, as observed for Rab4, Rab5, and Rab11 in other endomembrane systems (Sönnichsen et al., 2000).

Rab27b associates with distinct yet overlapping pools of SVs with Rab3a The quantitative and qualitative differences in SV localizations and membrane cycling dynamics between Rab3a and Rab27b indicate distinct yet overlapping roles for these regulatory GTPases in $\mathrm{Ca}^{2+}$ triggered exocytosis. This position is sup-

transfected neurons. $\boldsymbol{d}$, Expression of Rab27b mutants impairs SV cycling. Confocal images of axonal processes of cultured hippocampal neurons transfected with EGFP-Rab27b wildtype or mutant fusion proteins. The neurons were processed for FM4-64FX uptake (red) in the absence (control) or presence (stimulated) of electrical stimulation (600 APs, $20 \mathrm{~Hz})$ as detailed in the Materials and Methods. Scale bars, $5 \mu \mathrm{m}$. e, Bar graph summarizing FM4-64 uptake following transfection with the indicated vectors. The data were accumulated from separate cultures/transfections and expressed as the normalized means of analyzed fields \pm SEM $(n=40)$. 
ported by several complementary lines of evidence. First, the biochemical copurification and comigration of Rab27b and Rab3a on synaptic membranes imply coexistence on SV populations. Second, the specific targeting and colocalization of endogenous and exogenous Rab3a and Rab27b in synaptic nerve terminals confirms that they share common membrane transport pathways. Third, the coimmunoprecipitation of Rab27b with Rab3a-immunoisolated SVs strongly suggests that these two GTPases share interconnected SV pools. Fourth, the biochemical and morphological distinctions in Rab3a/Rab27b SV dissociation and recycling dynamics imply functionally divergent but complementary roles for these GTPases during $\mathrm{Ca}^{2+}$-triggered neurotransmitter release. Finally, the observations that Rab3a and Rab27a undergo differential membrane cycling dynamics (Handley et al., 2007) and coordinately regulate the docking of dense-core granules (Tsuboi and Fukuda, 2006) in neuroendocrine cells independently support overlapping but cooperative functions for Rab3a and Rab27b in exocytotic transport.

An unexpected finding in this study was that, unlike Rab3a, Rab27b possesses low cytosolic reserves and is remarkably resistant to GDI-mediated membrane extraction. While these observations are in general accord with the bulk of published reports indicating that Rab27a/b proteins are predominantly membrane bound (Zhao et al., 2002; Tiwari et al., 2003; Chen et al., 2004; Handley et al., 2007) and largely constitutively active (Kondo et al., 2006), they are difficult to reconcile with recent findings in parotid acinar cells whereby Rab27b was shown to redistribute from secretory granules to the cytosol following isoproterenol stimulation and exist in complex with Rab-GDI (Imai et al., 2009). The precise reason for this discrepancy remains unclear, but might point toward differences in cell systems and/or stimulation protocols used. On one hand, we were unable detect significant levels of Rab27b in Rab-GDI complexes or in presynaptic cytosol, suggesting that Rab27b remains membrane bound during SV recycling. Such a scenario is supported by recent data suggesting that GDP-Rab27b persists on membranes as an inactive homodimer that is not susceptible to GDI-mediated extraction (Chavas et al., 2007), and further strengthened by reports that prenylated Rab27 proteins undergo membrane targeting pathways distinct to that of classical REP1 and/or GDI-mediated delivery (Leung et al., 2007). On the other hand, we cannot exclude the possibility that Rab27b requires additional factors that were absent or in limited abundance in our in vitro assays. The Hsp90 chaperone complex, for example, has been shown to facilitate GDI-mediated Rab retrieval of Rab3a from synaptic membranes (Sakisaka et al., 2002). However, since Rab3a was efficiently retrieved from SVs in our extraction assays, additional as yet unidentified elements may be required for the recycling of Rab27b.

\section{Rab27b regulates the recycling of SVs}

By modulating the switch mechanism of Rab27b, we demonstrate, for the first time, that Rab27 proteins are functionally required for the recycling of SVs following $\mathrm{Ca}^{2+}$-triggered exocytosis at mammalian synapses. These findings complement recent observations in invertebrate systems, where recycling defects were noted following genetic ablation or antibody-induced depletion of Rab27 proteins (Mahoney et al., 2006; Yu et al., 2008). Interestingly, we found that both GTP- and GDP-preferring Rab27b mutants profoundly impair stimulus-dependent recycling of SVs. Similar effects have been observed using functionally equivalent mutants of other Rab GTPases, including the closely related Rab3 proteins (Schlüter et al., 2002; Star et al., 2005).

How does Rab27b regulate SV cycling during synaptic transmission? Considering the functional GTPase cycle of Rab pro- teins, Rab27b ${ }^{\text {Q78L }}$ may inhibit endogenous Rab27b function by sequestering or mislocalizing Rab27b effectors on SVs. Because of its high-affinity binding to GTP-dependent effectors and lack of GTPase activity, the Rab27b ${ }^{\text {Q78L }}$ mutant would thus be unable to disengage from tethering or docking factors that might be critical for SV fusion and/or recycling. In fact, several Rab27b effectors are known tethering factors and shown to contribute to SV cycling, including Slac2-c/MyRIP, Rabphilin, and RIM, the latter of which are also Rab3 effectors (Fukuda, 2008). In this sense, constitutively active Rab27b may sequester effectors shared with Rab3, making them unavailable for Rab3 function.

Conversely, expression of GDP-preferring forms of Rab27b likely exerts dominant-inhibitory effects on endogenous Rab27b function by sequestering Rab27- GEFs, thus resulting in the formation of "dead-end" complexes. This mechanism has been shown to underlie the dominant-negative effects of Rab and ras-related GTPases alike (Burstein et al., 1992; Chen et al., 2003). Given that Rab27 and Rab3 proteins have been recently shown to share common GEFs in both C. elegans (AEX3) and mice (Rab3GEF) (Mahoney et al., 2006; Figueiredo et al., 2008), it is likely that the expression of GDP-binding Rab27 mutants additionally compete out the endogenous Rab3 activation. The observed reductions in Rab3a immunofluorescence upon Rab27b ${ }^{\text {T23N/N133I }}$ overexpression would be consistent with this position. This might also account for the more pronounced synaptic defects observed in Rab3GEF and AEX3 deletions as compared single, double, and even quadruple knock-out models of Rab3 and Rab27 proteins (Geppert et al., 1997; Nonet et al., 1997; Schlüter et al., 2004; Mahoney et al., 2006; Gomi et al., 2007; Tolmachova et al., 2007). Although at this point in time the precise functional Rab3/Rab27b SV cycle requires further investigation, by analogy to other Rab3/ Rab27-expressing systems, an attractive model exists whereby Rab27b acts to "safeguard" the specificity of SV tethering or docking at release sites during SV cycling with functional diversity achieved through the rapid interswitching of divalent effectors between these two GTPases upon $\mathrm{Ca}^{2+}$-triggered Rab3 uncoupling. In this regard, the future characterization of double Rab3/Rab27b knock-out mice along with the identification of additional divalent Rab3/Rab27 effectors molecules may help to shed further light on the cooperative actions of these GTPases in synaptic transmission.

\section{References}

Babbey CM, Ahktar N, Wang E, Chen CC, Grant BD, Dunn KW (2006) Rab10 regulates membrane transport through early endosomes of polarized Madin-Darby canine kidney cells. Mol Biol Cell 17:3156-3175.

Bethani I, Lang T, Geumann U, Sieber JJ, Jahn R, Rizzoli SO (2007) The specificity of SNARE pairing in biological membranes is mediated by both proof-reading and spatial segregation. EMBO J 26:3981-3992.

Burstein ES, Brondyk WH, Macara IG (1992) Amino acid residues in the Ras-like GTPase Rab3A that specify sensitivity to factors that regulate the GTP/GDP cycling of Rab3A. J Biol Chem 267:22715-22718.

Castillo PE, Schoch S, Schmitz F, Südhof TC, Malenka RC (2002) RIMlalpha is required for presynaptic long-term potentiation. Nature 415:327-330.

Chavas LM, Torii S, Kamikubo H, Kawasaki M, Ihara K, Kato R, Kataoka M, Izumi T, Wakatsuki S (2007) Structure of the small GTPase Rab27b shows an unexpected swapped dimer. Acta Crystallogr D Biol Crystallogr 63:769-779.

Chen X, Ernst SA, Williams JA (2003) Dominant negative Rab3D mutants reduce GTP-bound endogenous Rab3D in pancreatic acini. J Biol Chem 278:50053-50060.

Chen X, Li C, Izumi T, Ernst SA, Andrews PC, Williams JA (2004) Rab27b localizes to zymogen granules and regulates pancreatic acinar exocytosis. Biochem Biophys Res Commun 323:1157-1162.

Chou JH, Jahn R (2000) Binding of Rab3A to synaptic vesicles. J Biol Chem 275:9433-9440. 
de Hoop MJ, Huber LA, Stenmark H, Williamson E, Zerial M, Parton RG, Dotti CG (1994) The involvement of the small GTP-binding protein Rab5a in neuronal endocytosis. Neuron 13:11-22.

Dejgaard SY, Murshid A, Erman A, Kizilay O, Verbich D, Lodge R, Dejgaard K, Ly-Hartig TB, Pepperkok R, Simpson JC, Presley JF (2008) Rab18 and Rab43 have key roles in ER-Golgi trafficking. J Cell Sci 121:2768-2781.

Figueiredo AC, Wasmeier C, Tarafder AK, Ramalho JS, Baron RA, Seabra MC (2008) Rab3GEP is the non-redundant guanine nucleotide exchange factor for Rab27a in melanocytes. J Biol Chem 283:23209-23216.

Fischer von Mollard G, SüdhofTC, Jahn R (1991) A small GTP-binding protein dissociates from synaptic vesicles during exocytosis. Nature 349:79-81.

Fischer von Mollard G, Stahl B, Khokhlatchev A, Südhof TC, Jahn R (1994a) Rab3C is a synaptic vesicle protein that dissociates from synaptic vesicles after stimulation of exocytosis. J Biol Chem 269:10971-10974.

Fischer von Mollard G, Stahl B, Walch-Solimena C, Takei K, Daniels L, Khoklatchev A, De Camilli P, Südhof TC, Jahn R (1994b) Localization of Rab5 to synaptic vesicles identifies endosomal intermediate in synaptic vesicle recycling pathway. Eur J Cell Biol 65:319-326.

Fukuda M (2008) Regulation of secretory vesicle traffic by Rab small GTPases. Cell Mol Life Sci 65:2801-2813.

Geppert M, Goda Y, Stevens CF, Südhof TC (1997) The small GTP-binding protein Rab3A regulates a late step in synaptic vesicle fusion. Nature 387:810-814

Gilchrist A, Au CE, Hiding J, Bell AW, Fernandez-Rodriguez J, Lesimple S, Nagaya H, Roy L, Gosline SJ, Hallett M, Paiement J, Kearney RE, Nilsson T, Bergeron JJ (2006) Quantitative proteomics analysis of the secretory pathway. Cell 127:1265-1281.

Gomi H, Mori K, Itohara S, Izumi T (2007) Rab27b is expressed in a wide range of exocytic cells and involved in the delivery of secretory granules near the plasma membrane. Mol Biol Cell 18:4377-4386.

Grosshans BL, Ortiz D, Novick P (2006) Rabs and their effectors: achieving specificity in membrane traffic. Proc Natl Acad Sci U S A 103: $11821-11827$.

Handley MT, Haynes LP, Burgoyne RD (2007) Differential dynamics of Rab3A and Rab27A on secretory granules. J Cell Sci 120:973-984.

Imai A, Yoshie S, Nashida T, Fukuda M, Shimomura H (2009) Redistribution of small GTP-binding protein, Rab27B, in rat parotid acinar cells after stimulation with isoproterenol. Eur J Oral Sci 117:224-230.

Jahn R, Scheller RH (2006) SNAREs—engines for membrane fusion. Nat Rev Mol Cell Biol 7:631-643.

Johnson JL, Brzezinska AA, Tolmachova T, Munafo DB, Ellis BA, Seabra MC, Hong H, Catz SD (2010) Rab27a and Rab27b regulate neutrophil azurophilic granule exocytosis and NADPH oxidase activity by independent mechanisms. Traffic 11:533-547.

Junutula JR, De Maziére AM, Peden AA, Ervin KE, Advani RJ, van Dijk SM, Klumperman J, Scheller RH (2004) Rab14 is involved in membrane trafficking between the Golgi complex and endosomes. Mol Biol Cell 15:2218-2229.

Khvotchev MV, Ren M, Takamori S, Jahn R, Südhof TC (2003) Divergent functions of neuronal Rab1 $1 \mathrm{~b}$ in $\mathrm{Ca}^{2+}$-regulated versus constitutive exocytosis. J Neurosci 23:10531-10539.

Kondo H, Shirakawa R, Higashi T, Kawato M, Fukuda M, Kita T, Horiuchi H (2006) Constitutive GDP/GTP exchange and secretion-dependent GTP hydrolysis activity for Rab27 in platelets. J Biol Chem 281:28657-28665.

Lang T, Jahn R (2008) Core proteins of the secretory machinery. Handb Exp Pharmacol:107-127.

Leung KF, Baron R, Ali BR, Magee AI, Seabra MC (2007) Rab GTPases containing a CAAX motif are processed post-geranylgeranylation by proteolysis and methylation. J Biol Chem 282:1487-1497.

Liu P, Bartz R, Zehmer JK, Ying YS, Zhu M, Serrero G, Anderson RG (2007) Rab-regulated interaction of early endosomes with lipid droplets. Biochim Biophys Acta 1773:784-793.

Mahoney TR, Liu Q, Itoh T, Luo S, Hadwiger G, Vincent R, Wang ZW, Fukuda M, Nonet ML (2006) Regulation of synaptic transmission by RAB-3 and RAB-27 in Caenorhabditis elegans. Mol Biol Cell 17:2617-2625.

Nonet ML, Staunton JE, Kilgard MP, Fergestad T, Hartwieg E, Horvitz HR, Jorgensen EM, Meyer BJ (1997) Caenorhabditis elegans rab-3 mutant synapses exhibit impaired function and are partially depleted of vesicles. J Neurosci 17:8061-8073.

Ostrowski M, Carmo NB, Krumeich S, Fanget I, Raposo G, Savina A, Moita CF, Schauer K, Hume AN, Freitas RP, Goud B, Benaroch P, Hacohen N,
Fukuda M, Desnos C, Seabra MC, Darchen F, Amigorena S, Moita LF, Thery C (2010) Rab27a and Rab27b control different steps of the exosome secretion pathway. Nat Cell Biol 12:19-30; sup pp 11-13.

Pereira-Leal JB, Seabra MC (2000) The mammalian Rab family of small GTPases: definition of family and subfamily sequence motifs suggests a mechanism for functional specificity in the Ras superfamily. J Mol Biol 301:1077-1087.

Pfeffer S, Aivazian D (2004) Targeting Rab GTPases to distinct membrane compartments. Nat Rev Mol Cell Biol 5:886-896.

Plutner H, Cox AD, Pind S, Khosravi-Far R, Bourne JR, Schwaninger R, Der CJ, Balch WE (1991) Rablb regulates vesicular transport between the endoplasmic reticulum and successive Golgi compartments. J Cell Biol 115:31-43.

Rizzoli SO, Betz WJ (2004) The structural organization of the readily releasable pool of synaptic vesicles. Science 303:2037-2039.

Sakisaka T, Meerlo T, Matteson J, Plutner H, Balch WE (2002) RabalphaGDI activity is regulated by a Hsp90 chaperone complex. EMBO J 21:6125-6135.

Schlüter OM, Schnell E, Verhage M, Tzonopoulos T, Nicoll RA, Janz R, Malenka RC, Geppert M, Südhof TC (1999) Rabphilin knock-out mice reveal that rabphilin is not required for rab3 function in regulating neurotransmitter release. J Neurosci 19:5834-5846.

Schlüter OM, Khvotchev M, Jahn R, Südhof TC (2002) Localization versus function of Rab3 proteins. Evidence for a common regulatory role in controlling fusion. J Biol Chem 277:40919-40929.

Schlüter OM, Schmitz F, Jahn R, Rosenmund C, Südhof TC (2004) A complete genetic analysis of neuronal Rab3 function. J Neurosci 24:6629-6637.

Shimizu H, Kawamura S, Ozaki K (2003) An essential role of Rab5 in uniformity of synaptic vesicle size. J Cell Sci 116:3583-3590.

Sönnichsen B, De Renzis S, Nielsen E, Rietdorf J, Zerial M (2000) Distinct membrane domains on endosomes in the recycling pathway visualized by multicolor imaging of Rab4, Rab5, and Rab11. J Cell Biol 149:901-914.

Star EN, Newton AJ, Murthy VN (2005) Real-time imaging of Rab3a and Rab5a reveals differential roles in presynaptic function. J Physiol 569: 103-117.

Stenmark H (2009) Rab GTPases as coordinators of vesicle traffic. Nat Rev Mol Cell Biol 10:513-525.

Südhof TC (2004) The synaptic vesicle cycle. Annu Rev Neurosci 27:509-547.

Takamori S, Riedel D, Jahn R (2000) Immunoisolation of GABA-specific synaptic vesicles defines a functionally distinct subset of synaptic vesicles. J Neurosci 20:4904-4911.

Takamori S, Holt M, Stenius K, Lemke EA, Grønborg M, Riedel D, Urlaub H, Schenck S, Brügger B, Ringler P, Müller SA, Rammner B, Gräter F, Hub JS, De Groot BL, Mieskes G, Moriyama Y, Klingauf J, Grubmüller H, Heuser J, et al. (2006) Molecular anatomy of a trafficking organelle. Cell 127:831-846.

Tisdale EJ, Balch WE (1996) Rab2 is essential for the maturation of preGolgi intermediates. J Biol Chem 271:29372-29379.

Tiwari S, Italiano JE Jr, Barral DC, Mules EH, Novak EK, Swank RT, Seabra MC, Shivdasani RA (2003) A role for Rab27b in NF-E2-dependent pathways of platelet formation. Blood 102:3970-3979.

Tolmachova T, Abrink M, Futter CE, Authi KS, Seabra MC (2007) Rab27b regulates number and secretion of platelet dense granules. Proc Natl Acad Sci U S A 104:5872-5877.

Tsuboi T, Fukuda M (2006) Rab3A and Rab27A cooperatively regulate the docking step of dense-core vesicle exocytosis in PC12 cells. J Cell Sci 119:2196-2203.

Ullrich O, Reinsch S, Urbé S, Zerial M, Parton RG (1996) Rab11 regulates recycling through the pericentriolar recycling endosome. J Cell Biol 135:913-924.

Wucherpfennig T, Wilsch-Bräuninger M, González-Gaitán M (2003) Role of Drosophila Rab5 during endosomal trafficking at the synapse and evoked neurotransmitter release. J Cell Biol 161:609-624.

Yu E, Kanno E, Choi S, Sugimori M, Moreira JE, Llinás RR, Fukuda M (2008) Role of Rab27 in synaptic transmission at the squid giant synapse. Proc Natl Acad Sci U S A 105:16003-16008.

Zerial M, McBride H (2001) Rab proteins as membrane organizers. Nat Rev Mol Cell Biol 2:107-117.

Zhao S, Torii S, Yokota-Hashimoto H, Takeuchi T, Izumi T (2002) Involvement of Rab27b in the regulated secretion of pituitary hormones. Endocrinology 143:1817-1824. 